\title{
Quantifying Quasar Variability As Part of a General Approach To Classifying Continuously Varying Sources
}

\author{
Szymon Kozłowski ${ }^{1}$, Christopher S. Kochanek ${ }^{1,2}$ \\ and \\ A. Udalski ${ }^{3}$, Ł. Wyrzykowski ${ }^{3,4}$ I. Soszyński ${ }^{3}$, M. K. Szymański ${ }^{3}$, M. Kubiak ${ }^{3}$, \\ G. Pietrzyński ${ }^{3,5}$, O. Szewczyk ${ }^{3,5}$, K. Ulaczyk ${ }^{3}$, R. Poleski ${ }^{3}$ \\ (The OGLE collaboration)
}

\begin{abstract}
Robust fast methods to classify variable light curves in large sky surveys are becoming increasingly important. While it is relatively straightforward to identify common periodic stars and particular transient events (supernovae, novae, microlensing events), there is no equivalent for non-periodic continuously varying sources (quasars, aperiodic stellar variability). In this paper we present a fast method for modeling and classifying such sources. We demonstrate the method using $\sim 86,000$ variable sources from the OGLE-II survey of the LMC and $\sim 2,700$ mid-IR selected quasar candidates from the OGLE-III survey of the LMC and SMC. We discuss the location of common variability classes in the parameter space of the model. In particular, we show that quasars occupy a distinct region of variability space, providing a simple quantitative approach to the variability selection of quasars.
\end{abstract}

Subject headings: cosmology: observations - galaxies: active — quasars: general - stars: variables: other

\footnotetext{
${ }^{1}$ Department of Astronomy, The Ohio State University, 140 West 18th Avenue, Columbus, OH 43210, USA; simkoz@astronomy.ohio-state.edu; ckochanek@astronomy.ohio-state.edu

${ }^{2}$ The Center for Cosmology and Astroparticle Physics, The Ohio State University, 191 West Woodruff Avenue, Columbus, OH 43210, USA

${ }^{3}$ Warsaw University Observatory, Al. Ujazdowskie 4, 00-478 Warszawa, Poland

${ }^{4}$ Institute of Astronomy, University of Cambridge, Madingley Road, Cambridge CB3 0HA, UK

${ }^{5}$ Universidad de Concepción, Departamento de Fisica, Casilla 160-C, Concepción, Chile
} 


\section{Introduction}

The focus of astronomy on the transient universe is steadily increasing because of the wealth of astrophysical information provided by time variability. Examples of past and current variability surveys are the Galactic microlensing surveys (e.g., OGLE, Udalski. Kubiak \& Szymański 1997; MOA, Sumi et al. 2003; MACHO, Alcock et al. 2000; EROS, Beaulieu et al. 1995; SuperMACHO, Becker et al. 2005), searches for both local (LOSS, Filippenko et al. 2001) and cosmologically distant (e.g., SDSS, Sako et al. 2008; CFHTLS, Cabanac et al. 2007; Essence, Miknaitis et al. 2007) supernovae, all sky variability surveys (e.g., ASAS, Poimański 1997; QUEST, Vivas et al. 2004; NSVS, Woźniak et al. 2004; Catalina, Drake et al. 2009) and prompt $\gamma$-ray burst monitors (e.g., BATSE, Meegan et al. 1992; RAPTOR, Vestrand et al. 2002; ROTSE, Rykoff et al. 2005; SWIFT, Burrows et al. 2005). A complete, or even partial, listing of more directed monitoring projects would be impossible, and the scale of variability surveys continues to grow rapidly with projects such as OGLE-IV (A. Udalski et al. 2010, in preparation), Pan-STARRS (Hodapp et al. 2004) and LSST (Ivezic et al. 2008).

A common problem for variability surveys is the classification of light curves given the enormous range of physical processes leading to variability (e.g., Sterken \& Jaschek 1996). Classification is well developed for simple periodic sources (e.g., Cepheids, RR Lyrae, eclipsing binaries, etc.) and impulsive (e.g., microlensing) or explosive sources (e.g., supernovae, novae), but less well developed for sources with non-periodic, continuous or very long timescale variability such as quasars. Most efforts at classification have focused on the broad spectrum of periodic sources, using the Fourier amplitudes of the light curves to recognize different variable classes using a broad range of methods (e.g., Eyer 2002; Pojmański 2002; Eyer \& Blake 2005; Soszyński et al. 2008b). More sophisticated methods for classification of variable objects include neural networks (e.g., Belokurov, Evans \& Le Du 2004) and selforganizing maps (e.g., Wyrzykowski \& Belokurov 2008).

Attempts at classifying non-periodic, continuously variable sources have largely focused on identifying quasars. Sesar et al. (2007) found that essentially all quasars vary at some level on long time scales, with $90 \%$ of Sloan Digital Sky Survey (SDSS) quasars having rms variability $\geq 0.03 \mathrm{mag}$ on a 6 year time scale. The average variability of quasars can be described by a structure function with greater variability amplitudes on longer time baselines (e.g., Vanden Berk et al. 2004; de Vries et al. 2005), and this has also been observed for smaller numbers of individually monitored quasars (e.g., Curti et al. 1985; Nelson 1996;

Collin et al. 2002; Sergeev et al. 2005). Thus, variability searches for quasars (e.g., Hawkins 1983; Veron \& Hawkins 1995, or Dobrzvcki et al. 2003a; Geha et al. 2003; Rengstorf et al. 2004a; Dobrzycki et al. 2005; Sumi et al. 2005 more recently) generally look for non-periodic sources with long time scale variability. The most formal approach to date is that used by 
Eyer (2002) and Sumi et al. (2005), where the selection criterion was based on the slope of the structure function of the light curves. These searches have generally been more successful in relatively empty extragalactic regions than in those with high stellar densities.

A significant problem in these attempts is that there was no simple method to reduce a non-periodic, continuously variable light curve to a small set of numbers, such as the periods and amplitudes of a Fourier series, which can be used for classification. Recently, Kelly et al. (2009) found that quasar light curves could be well modeled as a stochastic process with an exponential covariance matrix characterized by an amplitude and a time scale based on a small sample $(\sim 100)$ of quasars with multi-year light curves. Physically, the model is a damped random walk, and it has a broken power law structure function consistent with studies of quasar structure functions. Unfortunately, there are few large samples of quasars with extensive monitoring data. To our knowledge, there are the quasars in the SDSS equatorial strip, with roughly 60 epochs over $\sim$ six years (Sesar et al. 2007; Bramich et al. 2008), the QUEST survey (Rengstorf et al. 2004b), whose light curves are not publicly available, and any quasars lying in the microlensing survey regions. Recently, Kozłowski \& Kochanek (2009) used mid-IR color selection (e.g., Stern et al. 2005, also see Lacy et al. 2004) to identify a large sample of quasar candidates in the Magellanic Clouds. While they have yet to be spectroscopically confirmed, the nature of the selection method means that purity of the main sample of candidates should be high. By combining these candidates with the OGLEII and OGLE-III (Udalski, Kubiak \& Szymański 1997; Udalski et al. 2008) light curves, we can verify that quasar light curves are well described by a stochastic process based on a much larger sample of objects than used by Kelly et al. (2009) and then explore using this approach to light curve modeling for classifying light curves in general, including periodic sources. Obviously, a damped random walk is not an optimal model for periodic sources, but there is no technical difficulty in modeling them using the stochastic process.

This paper is organized as follows. In Section 2 we describe the light curve data and the existing catalogs of variable sources we can use for classifying light curves. In Section 3 we describe the mathematical model. We do not use the particular forecasting methodology of Kelly et al. (2009), but a more statistically optimal approach based on Press, Rybicki \& Hewitt (1992a) and Rybicki \& Press (1992, 1994) that we detail in the appendix. In Section 4 we examine how well the model works for the known quasars from Kelly et al. (2009) and the Kozłowski \& Kochanek (2009) quasar candidates, the distribution of quasars in the parameter space of the model and the completeness of variability selection. In Section 5 we examine the problem of contamination by determining the distribution of the general population of variable LMC sources in the model parameters. We also examine how the stochastic process models treat true periodic variables. In Section 6 we combine these results and discuss the problem of variability selecting quasars in dense stellar fields 
like the LMC. We summarize our results and discuss future uses in Section 7.

\section{Data}

The data used in this paper were collected with the $1.3 \mathrm{~m}$ Warsaw telescope at the Las Campanas Observatory, Chile during the second (1996-2000) and third (2001-2008) phases of the OGLE project (Udalski, Kubiak \& Szymański 1997; Udalski et al. 2008). The majority of the OGLE data were collected in the $I$-band $(\sim 370$ points per light curve in OGLE-III) with a smaller number of points in the $V$-band ( $\sim 40$ points per light curve). The OGLE-III light curves for many common variable stars are available on the internet 1 . The OGLE-II database of light curves ( $I$-band only) can be accessed through several web interface 2 (Szymański 2005; Udalski, Kubiak \& Szymański 1997). We make use of published OGLE samples of classical Cepheids (Cep; Soszyński et al. 2008b), double mode Cepheids (dCep; Soszyński et al. 2008a), RR Lyrae stars (RRLyr; Soszvński et al. 2009), OGLE Small Amplitude Variable Red Giants (OSARGs; Soszyński et al. 2004a), eclipsing binaries 3 (ECL; Wyrzykowski et al. 2003), ellipsoidal variable red giant.4 (ELL; Soszyński et al. 2004b), long secondary period variables (LSPs; Soszvński 2007), and long period variables (Miras, LPVs, and other semiregular variables, Soszyński et al. 2005) in the LMC. We separately extracted the OGLE-II light curves of Be stars from Keller et al. (2002), 300 OGLE-II and $\sim 2700$ OGLE-III light curves of the mid-IR selected quasar candidates from Kozłowski \& Kochanek (2009). We also reanalyzed the 109 quasars from Kelly et al. (2009).

In addition to these specific samples we downloaded $\sim 150,000$ "variable objects" from six OGLE-II LMC "inner" fields (SC1 - SC6) covering about $1.3 \mathrm{deg}^{2}$ and centered on the highest stellar density regions, and $\sim 38,000$ "variable objects" from the three lowest stellar density "outer" fields (SC15, SC19, and SC20). The core of this paper focuses on the analysis of the highest density inner fields as a worst case scenario for identifying quasars, with the lower stellar density outer LMC fields as a simpler comparison sample. An object was defined to be variable if the standard deviation of its light curve from a constant flux was at least twice the median photometric error (hereafter called the variability criterion). A large fraction of these "variables" are flat light curves with small numbers of outliers, and

\footnotetext{
${ }^{1}$ http://ogledb.astrouw.edu.pl/ ogle/CVS/

${ }^{2}$ http://ogledb.astrouw.edu.pl/ ogle/photdb/ and http://ogle.astrouw.edu.pl/ogle2/dia/

${ }^{3}$ http://ogle.astrouw.edu.pl/ogle2/lmc_ecl/

${ }^{4}$ http://ogle.astrouw.edu.pl/cont/4_main/var/ogleii/ell/ell.html
} 
these are removed by the following cleaning procedure. First, we correct the photometric errors following the procedure and values from Wyrzykowski et al. (2009). Then we removed up to five significant $(>5 \sigma)$ outliers (relative to their adjacent epochs) from each light curve, where a typical OGLE-II light curve has 360 epochs. After removing the sources no longer satisfying the variability criterion, we are left with 86,301 light curves in the inner LMC fields and 25,956 in the outer ones. The inner fields include $584 \mathrm{RR}$ Lyrae (499 "ab" and 85 "c"), 133 classical Cepheids, 7 double mode Cepheids, 6,745 OSARGs, 374 ellipsoidal variable red giants, 46 eclipsing binaries, 699 LSPs, 1,442 LPVs and 15 Be stars. We also use the 58 OGLE-II and 984 OGLE-III quasar candidates meeting the variability criterion.

On further inspection many of these "variable" stars are created by problems in the wings of the point spread functions (PSFs) of bright (variable) stars. This has been noted in earlier studies (e.g., Eyer 2002; Sumi et al. 2005). In particular, faint stars near bright variable stars tend to vary in phase with the bright source. We apply a three step procedure to remove these "ghost" variable objects. First, we mask regions near bright stars. Stars brighter than $I \approx 12.5 \mathrm{mag}$ are saturated in the OGLE data. These stars have PSF wings extending to 60 arcsec but they are not formally "detected" in the OGLE catalogs. In order to mask these stars, we used stellar objects from the 2MASS catalog (Cutri et al. 2003) brighter than $J=14$ mag. The masks are defined by rectangular regions with $\Delta$ R.A. $=2 \delta, \Delta$ decl. $=\delta$ around the Two Micron All Sky Survey (2MAss) position of each star, where $\delta=120(1-J / 14)$ arcsec. If a variable object is inside one of these regions, it is removed from the list of variables. We also masked visually identified regions which showed clear photometric problems (a dense clump of variable objects, variables detected on a "bloomed" CCD line, etc.). Our second criterion is that no variable could have any other variable object within 6 arcsec. Finally, the third criterion is that no variable can have a $>2$ mag brighter variable within 15 arcsec. These steps could be carried out with greater precision, but cleaning the OGLE catalog of spurious variables is not our primary focus even if it is ultimately a limitation and is one reason for using our rather conservative variability criterion. One additional problem with the OGLE-II photometry is a result of the realuminization of the telescope's mirror. A small number of light curves show a step-like change in magnitude between seasons 2 and 3 . These light curves are identified during the visual inspection we carried out for the final selection of quasar candidates. A summary of these photometric problems is also given in Ever (2002) and Sumi et al. (2005). 


\section{Methodology}

We are interested in modeling random processes leading to variability. Many such processes can be described by the covariance matrix $S$ of the signal, where we will be considering the particular case of an exponential covariance

$$
S_{i j}=\sigma^{2} \exp \left(-\left|t_{i}-t_{j}\right| / \tau\right)
$$

between epochs at $t_{i}$ and $t_{j}$ used by Kelly et al. (2009) to model quasar light curves based on a standard method from the forecasting literature (see, e.g., Brockwell \& Davis 2002). As explained in detail by Kelly et al. (2009), the physics of this process is a random walk driving term of amplitude $\sigma$ balanced by a damping time scale $\tau$ for a return to the mean. It is useful to define $\sigma^{2}=\tau \hat{\sigma}^{2} / 2$ and use $\tau$ and $\hat{\sigma}$ as our model parameters - on short time scales $|\Delta t|<<\tau$ the dispersion between two points is $\hat{\sigma}|\Delta t|^{1 / 2}$ while on long time scales it asymptotes to $\sigma$. Computationally, there is less covariance between $\tau$ and $\hat{\sigma}$ than $\tau$ and $\sigma$. The power spectrum of the process is

$$
P(f)=\frac{2 \hat{\sigma}^{2} \tau^{2}}{1+(2 \pi \tau f)^{2}}
$$

corresponding to white noise on long time scales $(f \rightarrow 0)$ and then a $\propto f^{-2}$ fall on short time scales $(f>1 / \tau)$. Kelly et al. (2009) suggest that $\tau$ may be related to the thermal time scale of accretion disks. We estimate these parameters using the approach of Press, Rybicki \& Hewitt (1992a), its generalization in Rybicki \& Press (1992) and the fast computational implementation in Rybicki \& Press (1994). We will collectively refer to these as the PRH method. In the appendix we summarize this approach, show how it can be used to derive the forecasting model of Kelly et al. (2009), and demonstrate that it is more statistically powerful than the forecasting approach while still requiring only $O(N)$ operations for a light curve with $N$ points.

Operationally, we fit a light curve by maximizing the likelihood (Equation (A8)) that the light curve can be fitted by the process model to determine the parameters $\tau$ and $\hat{\sigma}$ (as well as their uncertainties, if desired). We include the light curve mean as a simultaneously optimized linear parameter (Equations (A4) and (A7)). Given the estimates for $\tau$ and $\hat{\sigma}$, we have optimal estimates for the mean light curve at both the observed points (Equation (A3) ) and at any other time (Equation (A11)), as well as the variances about these means (Equations (A9) and (A12), respectively). Where we show these reconstructions and the "error snakes" defined by the variances, there are two important points to consider when comparing them to the data points. First, these variances are the variances in the mean light curve and not the variance of the data relative to the mean light curve. The latter

quantity, given in Equation (A10), is defined only where there is data and so is ill-suited to 
showing a continuous light curve. Data points will be scattered relative to the mean light curve by the combination of the variance in the mean light curve and the uncertainties in the individual data points. Second, the reconstructed light curve is not an example of an individual random walk defined by the parameters $(\tau$ and $\hat{\sigma})$, but rather the average of all such random walks that are consistent with the observed light curve given its uncertainties. The variance in the reconstructed light curve is then the variance of these individual random walks about the mean. If we generated individual random walks constrained by the data (see PRH), they would track the mean light curve and (statistically) stay inside the "error snake" defined by the variances, but they would show more structure on short time scales and excursions outside the "error snake" consistent with the estimated variances.

When we fit the model, we obtain a maximum likelihood estimate of $\tau$ and $\hat{\sigma}$ with $\log$ likelihood $\ln L_{\text {best }}$. There are two limiting cases for this model. First, as the time scale $\tau \rightarrow 0$, the covariance matrix becomes a diagonal matrix $S_{i j} \rightarrow \sigma^{2} \delta_{i j}$ equivalent to simply broadening the photometric errors. We refer to this limit as the "noise" limit with log likelihood $\ln L_{\text {noise }}$. We use a cut of $\ln L_{\text {best }}>\ln L_{\text {noise }}+2$ to select objects that are modeled by the exponential process. Given the variability criterion from Section 2, this cut mainly eliminates variable objects with $\tau$ smaller than the mean epoch separation. The other limit $\tau \rightarrow \infty$, with likelihood $\ln L_{\infty}$, is the limit where we cannot determine $\tau$ given the overall time span of the survey. This has not proved to be major issue with the OGLE data. We cannot distinguish $\tau$ from $\tau \rightarrow \infty$ for only $2 \%$ of the $\sim 86,000$ OGLE-II variables and $4.5 \%$ of the OGLE-III quasar candidates. For these objects the time scale $\tau$ was set to $\log _{10}(\tau) \simeq 5$.

We also check for periodicity in each light curve using the Lomb-Scargle periodogram method as implemented by Press et al. (1992b). For each light curve we note the three most likely periods $P_{i}(i=1,2,3)$ and their probabilities. We used narrow notches at $1,1 / 2,1 / 3$ and $1 / 4$ of a day to minimize diurnal aliasing problems, while still being able to examine RR Lyrae periods. These aliasing solutions could be improved, but this is not the focus of this paper. We classify a source as periodic if the estimated period $P$ is shorter than 200 days and the likelihood for the peak in the periodogram being observed at random is $\log _{10}\left(p_{\text {periodic }}\right)<-3$. We include a limit that periods must be shorter than 200 days because many quasars with large $\tau$ have a "period" satisfying $\log _{10}\left(p_{\text {periodic }}\right)<-3$ with $P>200$ day. These solutions corresponds to fitting a sine wave to the data, and as the number of time scales $\tau$ covered by the light curves shrinks, there is an increasing likelihood that a sine wave of some period will enormously improve the goodness of fit over no variability, leading to a false positive in the periodogram. For periodic variables we should see a strong correlation between $P$ and $\tau$. 


\section{Quasars}

For our quasar samples we use the 109 known quasars from Kelly et al. (2009) and $\sim 2,700$ mid-IR selected quasar candidates detected in the OGLE-III LMC and SMC fields from Kozłowski \& Kochanek (2009). The Kelly et al. (2009) sample is a heterogeneous mixture of MACHO variability selected quasars (Geha et al. 2003) and quasars monitored at least in part for reverberation mapping (Giveon et al. 1999; Peterson et al. 2004). They have the advantage of being confirmed quasars, but they are a non-random sample of quasars, and many of the reverberation mapping quasars have poorly sampled light curves compared to the MACHO or OGLE light curves. The mid-IR selected candidates have the disadvantage that they are not confirmed quasars. Kozłowski \& Kochanek (2009) classified the candidates based on their mid-IR colors (A/B for being inconsistent/consistent with a black body), location in the mid-IR color-magnitude diagram (CMD; YSO/QSO for whether the object is/is not in the region contaminated with young stellar objects, YSOs) and optical to mid-IR color (a/b for whether the optical to mid-IR colors are/are not consistent with spectroscopically selected quasars). Here we only use the "a" sources. Based on the extragalactic sources from the AGN and Galaxy Evolution Survey (AGES, C. S. Kochanek et al. 2010, in preparation), the QSO-Aa candidates, which are the majority, should be almost entirely comprised of quasars, while the other classes are likely dominated by contaminating sources.

Ideally, we should work with a quasar light curve that is free from contamination by the host galaxy. This can be a problem because we fit the light curves in magnitudes rather than flux (as did Kelly et al. 2009). In magnitude space, the effects of contamination on the process parameters are non-linear. Extra contaminating flux reduces amplitudes and increases time scales, while oversubtracting any contamination has the reverse effects. For the bright, distant quasars from Geha et al. (2003), Kozłowski \& Kochanek (2009) and, to a large extent, Giveon et al. (1999), this will be a limited problem. Not only are these very luminous quasars, but the observations are largely in the rest frame ultraviolet, where host galaxies have little flux. The Kozłowski \& Kochanek (2009) sample will also have few sources with significant host contamination because such sources are lost as part of the mid-IR selection (see Gorjian et al. 2008; Assef et al. 2009). It is emphatically not true of the local reverberation mapping targets. If we take the continuum light curves for MRK 279 (Santos-Lleó et al. 2001) , MRK 509 (Carone et al. 1996), NGC 3783 (Stirpe et al. 1994), NGC 4051 (Peterson et al. 2000), and NGC 5548 (Peterson et al. 2002, and references therein), and then either subtract the remaining host flux in the spectroscopic apertures or add in the host flux from outside the spectroscopic apertures based on Bentz et al. (2009), we see significant changes in the model parameters. On average, adding the rest of the host flux (subtracting the remaining host flux in the aperture) reduces (increases) the variability amplitude by an average of $-0.73 \mathrm{dex}(+0.38 \mathrm{dex})$ and increases (decreases) the characteristic time scale by an av- 
erage of +0.77 dex $(-0.42$ dex $)$ for five sources. The effects are larger when including the remainder of the host because the spectroscopic apertures used to obtain these light curves are already excluding most of the host galaxy flux - the host flux outside the aperture is 1-10 times larger than the host flux in the aperture. Thus, as with reverberation mapping, correlations of the process parameters with other quasar properties will be sensitive to the treatment of the host galaxy. We will not consider this problem further since we are focusing on the observed properties of quasar variability rather than their detailed interpretation.

Figure 1 shows four examples of QSO light curves modeled by the stochastic process. These fits are typical, and like Kelly et al. (2009), we find that most quasars are well modeled by the process, as illustrated by the $\chi^{2} / d o f$ distribution shown in Figure 2. Some care is required to interpret this distribution. All objects we consider are likely to be poorly described by no variability, so the process model will always produce a reduced $\chi^{2}$. Also keep in mind that one limit of the model is simply to broaden the error bars (the "noise" limit), which can always allow $\chi^{2} / d o f \rightarrow 1$ up to the effects of the priors on $\hat{\sigma}$ and $\tau$. Nonetheless, the final $\chi^{2} /$ dof distribution is far narrower than the distribution from fitting the linear model and few objects are doing so in the "noise limit" corresponding to simply broadening the uncertainties. The distribution is somewhat broader than it should be for correctly estimated Gaussian uncertainties. For a typical OGLE-III light curve with $N=360$ points, we expect $\chi^{2} / N \simeq 1 \pm \sqrt{2 / N}=1 \pm 0.07$. That the distribution is broader, and relatively symmetric, suggests that some of the difference lies in the accuracy of the photometric errors, since a $3 \%$ misestimation of the uncertainties is enough to cause such a shift. There is some skewness to larger $\chi^{2} / d o f$, but this could simply be due our pruning of outliers being too conservative - it only takes two $4 \sigma$ outliers to produce an 0.07 shift in $\chi^{2} / N$. Nonetheless, we cannot rule out the possibility that the process model is a poor or incomplete model for the variability physics in some objects.

The detection of variability is strongly magnitude-dependent because fainter sources require larger amplitudes for variability to be detected. Figure 3 shows the fraction of mid-IR quasar candidates that pass the two variability criteria based on either OGLE-II or OGLE-III data. One advantage of the process model is that we can explicitly calculate the completeness of a survey as a function of the parameters using Monte Carlo simulations. At each point on a grid of the process parameters $(\hat{\sigma}$ and $\tau$ ), we randomly generate $N$ light curves using the temporal sampling of a randomly selected real light curve. Generating simulated light curves of zero mean is trivial, since the next point

$$
s_{i+1}=s_{i} e^{-\Delta t / \tau}+G\left[\sigma^{2}\left(1-e^{-2 \Delta t / \tau}\right)\right],
$$

where $\Delta t$ is the time interval and $G\left(x^{2}\right)$ is a Gaussian deviate of dispersion $x$. The observed light curve is then $y_{i}=s_{i}+G\left(n_{i}^{2}\right)$ where $n_{i}$ is the observational noise. The chain is initiated 
by $s_{1}=G\left(\sigma^{2}\right)$. We use the mean measurement error at a given magnitude, but one could use the error estimates from a light curve matched in magnitude to the trial. Next, we apply our variability criterion, that the rms of the light curve must be twice the measurement errors, and that $\ln L_{\text {best }}>\ln L_{\text {noise }}+2$, to each trial light curve. The completeness is simply the fraction of the trials which pass both selection criteria. For speed, we estimate the second criterion by using the likelihood at the input parameters for $L_{b e s t}$ and the likelihood for the model with the same asymptotic variance but $\tau \rightarrow 0$ for $L_{\text {noise }}$.

Figure 4 shows the resulting completeness estimates for OGLE-II and OGLE-III light curves at $I=16.5,17.5,18.5$, and, $19.5 \mathrm{mag}$, where the typical photometric uncertainties (including the rescalings of Section 2) are $\sigma_{\text {phot }}=0.010,0.022,0.050$, and 0.12 mag for OGLEII and $\sigma_{\text {phot }}=0.008,0.017,0.037$, and 0.087 mag for OGLE-III. The general structure of the completeness limit is easily understood. The variability criterion can be approximated by noting that in the continuum limit, where we integrate over a continuous light curve rather examining discreet samples, the variance of a light curve described by an exponential covariance matrix relative to its mean is

$$
\operatorname{Var}(c(t))=\sigma^{2}\left[1-\frac{2}{x}+\frac{2}{x^{2}}(1-\exp (-x))\right],
$$

where $x=\Delta t / \tau$ is the ratio of the survey duration to the time scale $\tau$. When the survey duration is long compared to the time scale $(x \gg 1)$, the signal variance is simply $\sigma^{2}$, and light curves above the diagonal line with $\sigma^{2}+\sigma_{\text {phot }}^{2}>4 \sigma_{\text {phot }}^{2}$ will satisfy the variability criterion. When the survey is short $(x \ll 1)$, the observed variance is limited by the survey duration to $\sigma^{2} x / 3=\hat{\sigma}^{2} \Delta t / 6$ and the completeness is high if $\hat{\sigma}$ is sufficiently large. The criterion that the process is distinguishable from simply increasing the error bars eliminates sources in the corner with short time scales and high amplitudes. Note that the variability criterion is very conservative, because its selection limits do not improve with additional data. Using only the second criterion, based on whether the process model fits better than simply expanding the measurement errors, would lead to higher completeness for longer $\tau$ and smaller $\hat{\sigma}$ but at the expense of higher false positive rates due to systematic errors in the data. Compared to the OGLE-II survey, the OGLE-III survey is more sensitive and covers a longer temporal baseline but with a larger epoch spacing, so it is more complete for large $\tau$ and smaller $\sigma$, while less complete for small $\tau$. The completeness limit is largely determined by the variability criterion except for the corner with high amplitudes and short time scales. While not shown, the distribution of variable sources as a function of magnitude follows these completeness limits closely.

Figure 5 shows the distribution of quasars in an optical CMD, $\hat{\sigma}_{I}-\tau$ parameter space (shown in detail in Figure [6), $\hat{\sigma}_{I}$-magnitude space and, where available, the relationship between $\hat{\sigma}_{I}$ and $\hat{\sigma}_{V}$ at fixed $\tau$. Because the $V$-band light curves are poorly sampled compared 
to the $I$-band, we determine $\hat{\sigma}_{V}$ using the $\tau$ derived for the $I$-band light curve. To better understand the contamination problems we discuss later, we superpose a CMD of LMC stars based on the HST Local Group Stellar Photometry archive5 (Holtzman, Afonso \& Dolphin 2006). On the CMD one can quickly identify the red clump (RC), the red giant branch (RGB) and the top of the main sequence. Since these are derived from small Hubble Space Telescope (HST) fields, the sequences of higher luminosity stars are not significantly populated.

We see that quasars occupy a well defined locus in the $\hat{\sigma}-\tau$ space, lying in a band with $-2 \log _{10}(\hat{\sigma})+0.3 \leq \log _{10}(\tau) \leq-2 \log _{10}(\hat{\sigma})+1.6$ that roughly corresponds to an asymptotic variability of $\sigma \simeq 0.1 \mathrm{mag}$. Variability time scales longer than a few 1000 days are rare, although $\tau$ is indeterminate but long for about $5 \%$ of the sample. The estimate of $\tau$ becomes very uncertain as it approaches the survey length. At very short $\tau$, systems are lost because they fail the $\ln L_{\text {best }}>\ln L_{\text {noise }}+2$ criterion. If we focus on the QSO-A sources, which are overwhelmingly going to be real quasars, we can define a shaded region in Figure 5 (Cut 2 in Section (6) that encompasses most of these sources (69\% of those identified as variable in OGLE-III). The more contaminated QSO-B and YSO candidates are less likely to fall into this region (see Figure 6, Tables 1 and 2), providing added evidence of their higher levels of contamination by LMC sources. The mid-IR quasar candidates tend to lie in the Hertzsprung Gap of the CMD, consistent with the typical color $0.4 \leq(V-I) \leq 1.0$ mag of $z<3$ quasars. They also show relatively achromatic variability between the $V$ and $I$ bands, with more chromaticity for small $\hat{\sigma}$ and larger $\tau$ sources. Tables 1 and 2 summarize the properties of the mid-IR selected quasars for both OGLE-II and OGLE-III light curves. If we compare the locations of the quasars in the space of the variability parameters (Figure 66) to the completeness estimation of Figure 4, it is easy to understand the magnitude dependence of the fraction of variable quasars in Figure 3 .

The distribution of the quasars is a combination of intrinsic properties (variability at rest wavelength $\lambda$ for a black hole of some mass and luminosity), distance (magnitude and scalings between rest-frame and observed-frame properties), and selection effects (Figure 4). The observed magnitude of the quasar depends on distance and $K$-corrections, and the variability parameters have redshift corrections $\left(\tau_{\text {rest }}=\tau /(1+z)\right.$ and $\hat{\sigma}_{\text {rest }}=\hat{\sigma}(1+z)^{1 / 2}$ (Kelly et al. 2009)) and potentially $K$-corrections for any scalings of the parameters with wavelength, such as those seen in average structure functions (e.g., Vanden Berk et al. 2004).

\footnotetext{
${ }^{5}$ http://ganymede.nmsu.edu/lg - we selected two HST fields LMC_U2XQ05 and LMC_U2XQ06, each observed with the WFPC2 for 1540 and 1860 seconds in F555V and F814W, respectively. Since in both cases the PC1 chip was pointed at a globular cluster, we used the data from the three remaining WF chips, transformed to $V$ - and $I$-band. These data were then binned into $0.05 \mathrm{mag}$ bins in both magnitude and color and are shown as smoothed contours of 1, 5, 10 and 20 objects per bin in Figures [5] 7 and 8 .
} 
Our incompleteness depends on observed rather than intrinsic properties, and this makes it highly likely that our estimate of the region occupied by quasars is largely correct despite the increasing problems with completeness at fainter magnitudes. In essence, the majority of quasars which we lose due to incompleteness correspond to quasars with similar intrinsic properties at lower redshifts where they are bright enough to be included, rather than quasars with intrinsically different properties. The numbers of quasars observed to have a given set of parameters are, however, strongly dependent on the selection function, and reconstructing the true distribution will require redshifts and (essentially) a $V / V_{\max }$ method applied to the variability selection procedure. A significant advantage of our approach is that such problems can be quantitatively addressed using the variability model. The LMC sources we consider in the next section are an illustration of the opposite limit from the quasars. Since all LMC sources are at the same distance, we either detect them in this variability space given their magnitude or not.

We can also examine the distribution of the Kelly et al. (2009) quasars relative to the mid-infrared sample in Figure 6. They generally lie in the same region $(79 \%$ are in the selection box), but their distribution in that region is very different. Most of the MACHO and PG quasars lie at the large $\tau$ end of the distribution, with a few of the reverberation mapping quasars dribbling toward short $\tau$. The MACHO (Geha et al. 2003) quasars are variability selected, but the selection criterion may be a biased one compared to a (roughly) magnitude limited sampling of quasars. Figure 6 also shows the effects of adding or subtracting host galaxy discussed above.

\section{General Variability of Sources in the LMC}

In Section 4, we demonstrated that quasars occupy a limited region of the variability parameter space. In order to select quasars using variability, we need to examine how other variable sources populate these parameter spaces. In Figure 7, we show the location of the general variable populations from the inner LMC fields for the same combinations of variables used for quasars in Figure 5. The distributions of outer field variables are similar but of lower density. Where possible we have identified the variable type using the catalogs discussed in Section 2, Tables 1 and 2 summarize the statistics of selecting sources in these fields. It is critical to clean the input catalogs, since the variability criterion identifies

$\sim 86,000$ inner field variables before we apply the bright star masks, defect masks, and the isolation criteria described in Section 2, while there are only $\sim 13,000$ left afterward. Of these, $\sim 65,000$ (no masks) and $\sim 10,000$ (with masks) pass the restriction that the process model fits the data significantly better than simply expanding the photometric uncertainties 
(ln $\left.L_{\text {best }}>\ln L_{\text {noise }}+2\right)$. While the tables show the statistics for both cases, we only discuss the results for the sources including the masking and isolation criteria.

In Figure 7, we can quickly identify a number of sequences or overdensities of variable objects. We separate them, for clarity, in the four panels of Figures 8 12, In these figures we plot rare samples last so that they are not masked by the common ones. Quasars are shown in multiple panels to maximize their visibility compared to other populations, and Figures 5 and $[6$ are always available for reference. In the top-left panel of each figure we show two samples of OSARGs, where "a" corresponds to the asymptotic giant branch (AGB) objects and "b" to the RGB objects (Soszyński et al. 2007). They are approximately 3 mag brighter than the $\mathrm{RC}$ and also redder. In this panel, we also show slowly pulsating $\mathrm{B}$ stars (SPB, Kołaczkowski et al. 2006), active giants, active subgiants, and RS CVn binaries (AG and ASG, Drake 2006). There is no formal OGLE catalog of these variables, so we defined them based on the selection boxes shown in Figure 8. The OSARGs share the same area of the CMD with the LSPs, ELLs (top-right panel), and LPVs (bottom-right panel). The OGLE eclipsing binary samples are located just above the RC, and, slightly higher, one finds ellipsoidal variable red giants (top right). Along the instability strip with an approximate color of $(V-I)=0.5$, one can find Cepheids, $\sim 3$ mag brighter than RC, and RR Lyrae, which are slightly fainter then RCGs. The fundamental-mode Cepheids (RR Lyrae), denoted as "FU" ("ab"), and first-overtone ones, denoted as "FO" ("c"), form distinct sequences. Geha et al. (2003) in their attempt to select QSOs based on variability found that Be/Ae stars are the main source of contamination. Be stars can be found in the bottom-right panel just above the main sequence. In the bottom-left panel we present all the remaining variable objects. These should be quasars, other unclassified variables and sources with unidentified systematic problems. For comparison, we also show the OGLE-III quasar candidates in three of the panels, excluding the panel of unclassified sources.

The top-right panels of Figures 5 and 7 show the positions of objects in the "modified amplitude"-magnitude $(\hat{\sigma}-\mathrm{I})$ space. Once again, a number of source groupings are easily noticed, where the most prominent ones are OSARGs, LSPs, LPVs, RR Lyrae, and Cepheids (color coded in Figure 101). OSARGs, ELLs, LSPs, and LPVs overlap, although they can be partially distinguished based on their periods (Figure 12). In $\hat{\sigma}-\mathrm{I}$ space Cepheids and RR Lyrae occupy distinct areas.

We show the positions of all the variable stars and quasars in the space of the stochastic model parameters in Figures 5 and 7 (bottom-left panel). In this space of modified "characteristic time" and "variability amplitude" $(\tau-\hat{\sigma})$, we color coded all variability classes and present them in Figure 9, A majority of OSARGs and a substantial fraction of ELLs, LSPs, and LPVs can be easily distinguished from the quasar locus, although there is heavy 
contamination for long $\tau$. Note also that this is also the region occupied by most MACHO quasars, and this may be one cause of low yield of variability selected quasars in Geha et al. (2003). Cepheids, RR Lyrae, some LPVs, Be stars, ECLs, SPBs, and $\beta$ Cep, AGs and ASGs also overlap the quasar locus area. Note that fundamental mode and first overtone Cepheids and RR Lyrae can be distinguished from each other in $\hat{\sigma}-\tau$ space.

The bottom-right panels of Figures 5 and 7 show the variability ratio between the $V$ and $I$-bands as a function of $I$-band variability. The majority of LMC objects show higher variability amplitudes in the $V$-band than in the $I$-band, as we would expect since stellar variability is dominated by temperature changes. Quasar variability is less chromatic, so the amplitude ratio is another tool for separating quasars from variable stars. For these LMC fields, this is not critical because of the large magnitude differences between many of the variable stars and quasars. However, in a field dominated by Galactic sources spread over a wide range of distances, amplitude ratios have the advantage of being distance independent. The color-coded variability classes are shown in Figure 11.

Figure 12 shows all the variable sources and quasars (blue dots) in the space of the characteristic time-scale $\tau$ and the most likely period $P$ derived from a standard periodogram. As noted in Section 3, quasars never have significant probabilities of being periodic sources unless the "period" is long, $P \gtrsim 200$ days. On inspection, these light curves have fluctuations on long time scales that are quasi-periodic over the extent of the light curve (particularly with yearly or half-yearly aliases), so a sine wave fit to the data is a significantly better fit to the data than a constant. Even the quasars with falsely probable periods show no correlation between the period and $\tau$. This leads us to define a periodic source as one with $P<200$ days and $\log _{10}\left(p_{\text {periodic }}\right)<-3$. On short time scales $(\tau$ or $P \lesssim 1$ day) we see no real correlations because the periodograms have diurnal aliasing problems and many sources fail to pass the $\ln L_{b e s t}>\ln L_{n o i s e}+2$ variability selection criterion. For periods of days to tens of days, many sources follow a scaling with $\tau \propto P^{2}$, which is surprising since we expect $\tau \propto P$ by dimensional analysis.

Our one hypothesis for this scaling is that it can be produced if the exponential process model is dominated by how well it fits the peak of the correlation function. Near the peak, the auto-correlation function of a sine wave is proportional to $\Delta t^{2} / P^{2}+$ const, while that of the exponential covariance matrix is $\Delta t / \tau+$ const, potentially driving the $\tau \propto P^{2}$ scaling. There is a regime where the longer period Cepheids have $\tau \propto P$, and some of the multiperiodic sources (OSARGs) appear in multiple groupings. Monte Carlo experiments fitting sine wave light curves show that the relation between $\tau$ and $P$ is strongly affected by the temporal sampling, with the $\tau \propto P^{2}$ region shifting to longer periods as the mean epoch spacing increases. Despite some effort we could not derive an analytical model for the scaling. It 
is clear, however, that the relationship between $\tau$ and $P$ depends on sampling rather than being universal.

\section{Variability Selection of Quasars}

We can now combine the results from Sections 4 and 5 to define selection criteria for isolating quasars from other variable sources. Presently this is limited to searching for candidate quasars in the $1.3 \mathrm{deg}^{2}\left(0.65 \mathrm{deg}^{2}\right)$ covered by the OGLE-II inner (outer) fields in order to explore how well we can find quasars while avoiding contamination. We limit the analysis to sources with $I<19.5$ based on the completeness estimates in Section 4 . There should be very few quasars in this region, so this is really a test of contamination levels in a field with very high densities of stellar variables. Based on Richards et al. (2006) we expect approximately 1, 6, and 20 quasars per square degree with $I<17.5$, 18.5, and 19.5 mag respectively, which agrees well with the statistics of the mid-IR selected quasars (Kozłowski \& Kochanek 2009). We will simultaneously apply the selection method to the broader sample of OGLEII and OGLE-III quasar light curves to examine their effects on completeness. We select quasars in four steps, starting with two that will be generic to any set of light curves from any location (Cut 1 - non-periodicity, Cut 2 - variability properties of quasars), and then applying those that depend on the specific field (Cut 3) and the availability of light curves in multiple bands (Cut 4). We will not apply a color selection criterion, since one goal of any wide application of this method would be to find quasars at redshifts where their colors are crossing the stellar color distribution near $z \sim 2.6$ (Richards et al. 2006).

Cut 1. We start by eliminating periodic sources. A periodic source is defined to have a maximum likelihood period $P<200$ days with $\ln \left(p_{\text {periodic }}\right)>-3$. This removes $76 \%$ of the sources, including, $99 \%$ of RR Lyrae, $100 \%$ of Cepheids, $91 \%$ of ELLs, and $100 \%$ of ECLs. It does not remove $20 \%$ of OSARGs, $69 \%$ of LSPs, and $36 \%$ of LPVs.

Cut 2. Next we isolate sources with the variability properties of quasars, defined by the region in $\hat{\sigma}-\tau$ space bounded by $-2 \log _{10}(\hat{\sigma})+0.3<\log _{10}(\tau)<-2 \log _{10}(\hat{\sigma})+1.6$, $\log _{10}(\hat{\sigma})>-1.1$ and $\tau>2$ days. This quasar region is shown in gray in Figures 4, 5, 6, 7 and 9. The lower limits are designed to remove most Cepheids, RR Lyrae, OSARGs, ellipsoidal variable red giants, long secondary period variables, slowly pulsating $\mathrm{B}$ stars, $\beta$ Cephei stars, active giants and subgiants, and YSO-(AB) objects. The upper limits remove many of the long period and long secondary period variables. This criterion will still leave significant contamination from several of the variable star populations along the left (low $\hat{\sigma})$ and bottom edges. These are blue variables, active giants and subgiants and RR Lyrae. This cut removes $97 \%$ of ELLs, $92 \%$ of OSARGs, $45 \%$ of LSPs, and $41 \%$ of LPVs passing 


\section{Cut 1.}

Cut 3. The third cut takes advantage of the fact that almost all the contaminating sources are at a common distance and largely consist of relatively bright giant stars. It is defined by a wedge in $\hat{\sigma}$-magnitude space that isolates the OGLE-III quasars. We first require quasar candidates to be fainter than $I=16$ mag since such bright quasars are very rare, while the OSARGs, classical Cepheids, eclipsing binaries, ellipsoidal variable red giants and long period variables are all brighter than $I=16$ mag given the distance to the LMC. A second limit, $I>3 \log _{10}(\hat{\sigma})+18.5$, separates the RR Lyrae, double mode Cepheids, ECLs, some ELLs, SPBs, $\beta$ Cep, AGs and ASGs, which are to the right of the quasar region. As in Cut 1 , we use the same lower limit on the scaled amplitude of $\log _{10}(\hat{\sigma})>-1.1$, which partially eliminates Be stars. This cut removes $100 \%$ of the ELLs, LPVs, LSPs and OSARGs passing Cut 2. Cut 3 would need to be adjusted for other background environments, such as the Galactic bulge, where similar variable stars are $\sim 4$ mag brighter than in the LMC (depending on the amount of extinction).

Cut 4. For the objects with well determined $V$-band amplitudes $\hat{\sigma}_{V}$ we can also separate quasars using the ratio of the $V$ - and $I$-band amplitudes. We define an area in $\left(\hat{\sigma}_{V} / \hat{\sigma}_{I}\right)-\hat{\sigma}_{I}$ space with $-1.1<\log _{10}\left(\hat{\sigma}_{I}\right)<0.6$ and $\log _{10}\left(\hat{\sigma}_{V} / \hat{\sigma}_{I}\right)<-0.2 \log _{10}\left(\hat{\sigma}_{I}\right)+0.2$, where we select objects with a smaller ratio of the $V$ - to $I$-band variability amplitudes than is typical for stars (Figure 11, gray area). This cut removes most Cepheids and RR Lyrae and a substantial fraction of other common variable stars.

The effects of the selection functions on both the LMC variable population and these quasar samples are summarized in Tables 1 and 2 both for their effects when applied sequentially (Table 1) and individually (Table 2). We also summarize the effects on the subcategories of the mid-IR selected samples, where we expect to eliminate more of the heavily contaminated QSO-B and YSO classes than the relatively high purity QSO-A class. Recall from Section 4 that we lose a large fraction of the quasars from the two requirements for being considered a variable because most quasars are relatively faint compared to the depths of the OGLE data.

The first test of a selection method is its completeness. We evaluate this based on the on the $\sim 2,700(\sim 300)$ mid-IR selected quasar candidates from Kozłowski \& Kochanek (2009) with OGLE-III (OGLE-II) light curves as well as the smaller Kelly et al. (2009) sample. The biggest problem for variability selected quasars from these samples is that most quasars will be faint. While we can identify 2716 (310) of our mid-IR quasars in the OGLE-III (OGLE-II) data, only 1139 (215) are bright enough $(I<19.5 \mathrm{mag})$ to plausibly detect quasar variability (see Figure 3). For the full OGLE-III (OGLE-II) samples, 984 (58) pass

the basic variability criterion of Section 2 , while 635 (58) of the $I<19.5$ mag candidates 
pass this criterion. Adding the noise criterion, $\ln L_{\text {best }}>\ln L_{\text {noise }}+2$, we find 619 (848) for OGLE-III and 55 (55) for OGLE-II, brighter than $I<19.5$ mag (no magnitude limit). The difference in the fraction of variable objects in the two phases of the OGLE survey is explained by the added depth (0.3 mag) and duration (4 versus 8 years) of the OGLE-III survey. By design, the remaining cuts have little effect on the quasar candidates, although we lose a larger fraction of the non-QSO-A sub-samples, as we would expect given their higher levels of contamination. After applying the first three cuts to the variable objects, we are left with $65 \%$ of the QSO-A sample. We can apply only Cuts 1 and 2 to the Kelly et al. (2009) sample, and $65 \%$ of the sources pass the cuts. Cut 4 removes an additional $7 \%$ of the OGLE-III QSO-Aa objects. Finally, all four cuts are met by $61 \%(63 \%)$ of the OGLE-III QSO-Aa quasar candidates with no faint magnitude limit (within $16<I<19.5 \mathrm{mag}$ ) and $17 \%$ of the OGLE-II candidates. Thus, following this approach can yield variability-selected samples of quasars with moderate completeness.

Next we examine how well we reject other variable sources, focusing on our analysis of the central region of the LMC. We only discuss the sample including masks and isolation criteria (see Section 2), but report the results for the full, unpruned sample as well in Tables 1 and 2. The selection cuts are very effective at reducing the numbers of candidate sources, going from $\sim 10,000(\sim 600)$ sources passing the variability criterion in the inner (outer) fields to only 58 (2) candidate quasars. In the outer fields with fewer stars, the contamination is remarkably low.

Of the 58 remaining OGLE-II candidates in the inner fields, 3 are also mid-IR selected quasars. There are 63 (41 QSO-A) OGLE-II sources in the same fields, 24 (12) passing the two initial variability criteria, showing that the biggest problem for quasars is recognizing variability given the depth of the data. The mid-IR quasar selection method is not perfect, in particular it tends to miss lower redshift quasars with significant mid-IR emission from the host galaxy (Goriian et al. 2008; Assef et al. 2009), so some of the remaining, unidentified candidates may be real quasars. For the Richards et al. (2006) quasar number counts, we expect of order 1, 8, and 25 quasars brighter than $I<17.5,18.5$ and, 19.5 mag in this $1.3 \mathrm{deg}^{2}$ region, compared to 6, 16 and 41 QSO-Aa candidates in OGLE-II. We examined the light curves of all 58 objects that passed the four criteria. On visual inspection, the majority seem to be created by photometric problems in the wings of other stars, long period variable stars just above the RCG, stars in the wings of high proper motion stars (see Ever \& Woźniak 2001), blue and Be stars, eruptive variables, novae/supernovae, nova-like variable, unidentified types of variable stars, and quasar candidates. Of the non-mid-IR sources, only one seems likely to be a quasar, the OGLE-II (OGLE-III) object LMC_SC5 253536 (LMC167.2 36755, at J2000 05:24:24.17 -69:55:56.9). It has a brightness of $I=18.53$ (18.37) mag in OGLE-II (OGLE-III). Its color of $(V-I)=1.38$ (1.06) mag is somewhat 
redder than expected for $z<3$ quasars. We show both the OGLE-II and OGLE-III light curves in Figure 13 .

In the outer OGLE-II fields we are left with only 2 candidates. On inspection we find no plausible QSO candidates. None of the OGLE-II light curves of the 43 mid-IR selected candidates (29 QSO-Aa) from the outer fields passes all the criteria. In fact, only two QSO-Aa and two YSO-Aa objects with OGLE-II light curves pass the two initial variability criteria.

\section{Summary}

Periodograms to recognize periodically varying sources and begin their classification are a standard astronomical tool. Here we introduce an equally simple approach to classifying continuously varying sources that are not periodic and explored the properties of large samples of variable sources in the LMC and SMC using data from the OGLE-II and OGLE-III microlensing projects. We modeled the sources as a damped random walk, a stochastic process with an exponential covariance matrix characterized by a scaled amplitude $\hat{\sigma}$, a time scale $\tau$, and an asymptotic variance on long time scales of $\sigma=\hat{\sigma}(\tau / 2)^{1 / 2}$. This model was introduced by Kelly et al. (2009), who used a forecasting approach to estimate the parameters that is not as statistically powerful as the PRH method used here. Extracting the damped random walk parameters is no more complex than obtaining a periodogram, so it can be easily used to characterize any light curve.

We first applied this method to a much larger sample of quasars than Kelly et al. (2009) by using the mid-IR selected quasars from Kozłowski \& Kochanek (2009) that also have OGLE-II or OGLE-III light curves. We have verified the suggestion by Kelly et al. (2009) that quasar light curves are well modeled by the damped random walk process. Moreover, we find that quasars occupy a well defined region of the parameter space of the model, allowing us to design a simple quantitative method for the variability selection of quasars. The details of the approach will likely require some modifications as larger samples of quasars are analyzed and the method is applied in regions with different sources of stellar contamination, but the broad outline should survive. We also note that the model allows quantitative estimation of completeness in variability selected quasar samples and can estimate the detection ranges needed for computing luminosity functions or other population statistics.

While Kelly et al. (2009) are certainly correct that a damped random walk corresponding to an exponential covariance matrix models quasar variability well, it is less clear that the correlations between the model parameters and the luminosity or black hole masses of 
quasars are robust. Since we lack redshifts for our quasar sample we cannot explore this in detail, but we note two potential systematic problems in the Kelly et al. (2009) analysis. First, the distribution of the Kelly et al. (2009) quasar sample may be a biased sampling of the quasar distribution. It appears to be biased toward sources with long time variability time scales compared to the mid-IR quasar sample. Second, most of the leverage for determining the slopes in the relations between $\tau$ and $\hat{\sigma}$ and the quasar mass and luminosity in Kelly et al. (2009) come from the relatively low black hole mass and luminosity reverberation mapping quasars. Host galaxy contamination is a major issue for these systems (Bentz et al. 2009), and we find that this also holds for estimates of the variability process parameters because light curves in magnitudes are non-linearly distorted by contamination. Thus, the slopes of the Kelly et al. (2009) correlations may strongly depend on the treatment of these hosts. Just as for the reverberation mapping studies, we should really measure the variability parameters of the quasar with no diluting contribution from the host galaxy, but this is virtually impossible to do for large samples. This problem could be avoided by modeling the time variable flux rather than modeling the time varying magnitudes, but the meaning of $\hat{\sigma}$ then becomes strongly distance dependent and fractional variability, as represented by variability in magnitudes, probably has greater physical meaning. These issues are not a concern for simply classifying light curves.

We developed a set of selection cuts to try to identify quasars based on their variability, and $63 \%(17 \%)$ of the variable quasars in the QSO-A sources in the OGLE-III (OGLE-II) surveys pass the selection criteria. In a lower stellar density field this can be improved by using more liberal selection criteria. If we apply the same cuts to the 10,000 (600) OGLE-II variable sources in these high density inner (lower density outer) fields, only 58 (2) candidates survive, of which 3 (0) are mid-IR selected quasar candidates. After visual inspection, only $1(0)$ of the other sources is a quasar candidate. Most of the rest are artifacts that could be avoided by better pruning the input catalogs. The remaining sources are blue and eruptive variables, variable giants and supergiants. While 85-93\% contamination (depending on how we count remaining artifacts) seems poor, it was achieved when searching for rare extragalactic sources in an extremely high density stellar field $\left(\sim 10^{6}\right.$ stars and $\sim$ 8,000 variable stars $/ \mathrm{deg}^{2}$ compared to $\sim 20$ quasars $/ \mathrm{deg}^{2}$ with $16 \leq I \leq 19.5 \mathrm{mag}$ ), and the bulk of the false positives were easy to recognize by visual inspection. If we add a color cut for $z<3$ quasars $(0.4 \leq(V-I) \leq 1.0 \mathrm{mag})$ we can also greatly reduce the residual contamination. In the outer LMC fields $\left(\sim 10^{5}\right.$ stars and $\sim 10^{3}$ variable stars $\left./ \mathrm{deg}^{2}\right)$ or an extragalactic field $\left(>10^{3}\right.$ stars and a small number of variable stars $\left./ \mathrm{deg}^{2}\right)$ there will be little difficulty with contamination. Moreover, it is clear that the OGLE-II data are too shallow to search for quasars in small areas compared to OGLE-III.

Clearly the next steps are to apply this approach systematically. Here we carried out 
a limited analysis of OGLE-II and OGLE-III data pending the general availability of the full sample of OGLE-III light curves. We are also in the process of obtaining redshifts for many of the mid-IR quasar candidates. An obvious next step is to characterize the entire OGLE-III survey. In C. L. MacLeod et al. (2010, in preparation), we analyze the nearly 9,000 known quasars with light curves from the SDSS to explore the correlations found by Kelly et al. (2009). These SDSS data can also be used to explore the problems of background sources and their classification for typical extragalactic fields rather than the center of the LMC.

We thank Brandon Kelly for answering questions and generously supplying both his original IDL code and the light curves used in his study. We also thank George Rybicki for consulting on aspects of the fast implementation of his method. We thank R. J. Assef for providing us with the colors of quasars at various redshifts, and the anonymous referee, whose comments helped us to improve the manuscript. This work has been supported by NSF grant AST-0708082. The OGLE project is partly supported by the Polish MNiSW grant N20303032/4275.

\section{A. The PRH Method}

The basic idea (Press, Rybicki \& Hewitt 1992a; Rybicki \& Press 1992, 1994) is to suppose that we have data $\mathbf{y}$ that is due to an underlying, true, signal $\mathbf{s}$, measurement noise at each point $\mathbf{n}$, and a general trend defined by a response matrix $L$ and a set of linear coefficients $\mathbf{q}$, thus, $\mathbf{y}=\mathbf{s}+\mathbf{n}+$ Lq. For example, we will use the linear coefficients to optimally remove the light curve mean, so we have one linear coefficient $q_{1}$ for the mean, and the response matrix is simply a column vector $L_{i 1}=1$ with an entry for each data point, $i=1 \cdots N 6$ The intrinsic variability has covariance matrix $S=\langle\mathbf{s s}\rangle$ and the noise has covariance matrix $N=\langle\mathbf{n n}\rangle$. We will be explicitly relying on Gaussian statistics in order to add normalizing pre-factors to the squared difference statistics focused on by PRH. By definition, we know that

$$
P(\mathbf{s}) \propto|S|^{-1 / 2} \exp \left(-\frac{\mathbf{s}^{T} S^{-1} \mathbf{s}}{2}\right) \quad \text { and that } \quad P(\mathbf{n}) \propto|N|^{-1 / 2} \exp \left(-\frac{\mathbf{n}^{T} N^{-1} \mathbf{n}}{2}\right) .
$$

\footnotetext{
${ }^{6}$ Additional uses of the linear parameters follow trivially. To remove a linear trend, $q_{1}+q_{2}\left(t-t_{0}\right)$, from the light curve, we simply add a second column $L_{i 2}=t_{i}-t_{0}$ to the response matrix. If we have two light curves with a possible offset, we could use separate means $q_{1}$ and $q_{2}$ for each segment, and then $\left(L_{i 1}, L_{i 2}\right)=(1,0)$ for data in segment 1 and $\left(L_{i 1}, L_{i 2}\right)=(0,1)$ for data in segment 2 .
} 
Thus, the probability of the data given the linear coefficients $\mathbf{q}$, the intrinsic light curve $\mathbf{s}$, and any other parameters of the model p (e.g. $\tau$ and $\hat{\sigma}$ ) is

$$
P(\mathbf{y} \mid \mathbf{q}, \mathbf{s}, \mathbf{p}) \propto|S N|^{-1 / 2} \int d^{n} \mathbf{n} \delta(\mathbf{y}-(\mathbf{s}+\mathbf{n}+L \mathbf{q})) \exp \left(-\frac{\mathbf{s}^{T} S^{-1} \mathbf{s}+\mathbf{n}^{T} N^{-1} \mathbf{n}}{2}\right) .
$$

After evaluating the Dirac delta function, we complete the squares in the exponential with respect to both the unknown intrinsic source variability $\mathbf{s}$ and the linear coefficients $\mathbf{q}$. This exercise determines our best estimate for the mean light curve,

$$
\hat{\mathbf{s}}=S C^{-1}(\mathbf{y}-L \hat{\mathbf{q}})
$$

and the linear coefficients,

$$
\hat{\mathbf{q}}=\left(L^{T} C^{-1} L\right)^{-1} L^{T} C^{-1} \mathbf{y} \equiv C_{q} L^{T} C^{-1} \mathbf{y}
$$

where $C=S+N$ is the overall covariance matrix of the data and $C_{q}=\left(L^{T} C^{-1} L\right)^{-1}$. With these definitions we can factor the argument of the exponential into

$$
P(\mathbf{y} \mid \mathbf{q}, \mathbf{s}, \mathbf{p}) \propto|S N|^{-1 / 2} \exp \left(-\frac{\boldsymbol{\Delta s}^{T}\left(S^{-1}+N^{-1}\right) \boldsymbol{\Delta} \mathbf{s}}{2}-\frac{\Delta \mathbf{q}^{T} C_{q}^{-1} \boldsymbol{\Delta} \mathbf{q}}{2}-\frac{\mathbf{y}^{T} C_{\perp}^{-1} \mathbf{y}}{2}\right)
$$

where

$$
C_{\perp}^{-1}=C^{-1}-C^{-1} L C_{q} L^{T} C^{-1}
$$

is the component of $C$ that is orthogonal to the fitted linear functions, the variances in the linear parameters are

$$
\left\langle\Delta \mathbf{q}^{2}\right\rangle=\left(L^{T} C^{-1} L\right)^{-1} \equiv C_{q},
$$

$\Delta \mathbf{s}=\mathbf{s}-\hat{\mathbf{s}}$ and $\Delta \mathbf{q}=\mathbf{q}-\hat{\mathbf{q}}$. We can marginalize the probability over the light curve $\mathbf{s}$ and the linear parameters $\mathbf{q}$ under the assumption of uniform priors for these variables to find that

$$
P(\mathbf{y} \mid \mathbf{p}) \propto|S+N|^{-1 / 2}\left|L^{T} C^{-1} L\right|^{-1 / 2} \exp \left(-\frac{\mathbf{y}^{T} C_{\perp}^{-1} \mathbf{y}}{2}\right),
$$

where for the exponential model the remaining parameters $\mathbf{p}$ are $\tau$ and $\hat{\sigma}$. This is the likelihood we optimize to determine $\tau$ and $\hat{\sigma}$. We also know the variances in the estimate for the mean light curve

$$
\left\langle\Delta \mathbf{s}^{2}\right\rangle=S-S^{T} C_{\perp} S
$$

and the variance between the data and the estimated light curve is

$$
\left\langle(\mathbf{y}-\hat{\mathbf{s}}-L \hat{\mathbf{q}})^{2}\right\rangle=N\left(1-C^{-1} C_{q}^{-1}\right) C^{-1}\left(1-C_{q}^{-1} C^{-1}\right) N
$$


Our only addition here compared to $\mathrm{PRH}$ is keeping track of the normalizing prefactor of the exponential.

The relation to Kelly et al. (2009) comes from using this formalism to predict the value of the time series at an unmeasured time. The simplest means of doing so is simply to pad the data vector $\mathbf{y}_{d}$ with additional fake points $\mathbf{y}_{f}$ that have infinite measurement uncertainties in the sense that $N^{-1} \rightarrow 0$ for these points. For simplicity we consider the case without additional linear parameters. We partition the signal and noise matrices as $S_{d d}, S_{f f}, S_{d f}$ and $S_{d f}$ and $N_{d d}^{-1}, N_{f f}^{-1}=0, N_{d f}^{-1}=0$ and $N_{d f}^{-1}=0$ for the data-data, fake-fake, data-fake, and fake-data blocks of the matrices. Substituting into Equation (A3), we find that the estimate of the true light curve for the measured data points is the result we would obtain without having padded the data vector, and that the estimate of the light curve at the additional points is

$$
\hat{\mathbf{s}}_{f}=S_{f d}\left(S_{d d}+N_{d d}\right)^{-1} \mathbf{y}_{d}
$$

with variance relative to the mean light curve of

$$
\left\langle\boldsymbol{\Delta}_{f}^{2}\right\rangle=S_{f f}-S_{f d}\left(S_{d d}+N_{d d}\right)^{-1} S_{d f}
$$

These two expressions define the mean light curves and the "error snakes" shown in Figures 1 and 13. Mathematically, the mean light curve is the weighted average of all process light curves described by parameters $\mathbf{p}$ that are statistically consistent with the data, and the variance is the scatter of these light curves about this mean.

We can use these results to "forecast" the light curve by noting that we can express the expected value at epoch $i+1$ in terms of the measurement at $i$ and the forecast for $i$ based on the $i-1$ earlier data points. In component language,

$$
\hat{s}_{i}=S_{i j}(S+N)_{j k}^{-1} y_{k}
$$

where $j, k=1 \cdots i-1$, and

$$
\hat{s}_{i+1}=S_{i+1 j}(S+N)_{j k}^{-1} y_{k}
$$

where $j, k=1 \cdots i$. For an exponential covariance matrix, $S_{i+1 j}=\alpha S_{i j}$ where $\alpha=$ $\exp \left(-\left|t_{i+1}-t_{i}\right| / \tau\right)$. If you partition the $S+N$ matrix of Equation (A14) into the $i-1 \times i-1$ part used to estimate $\hat{s}_{i}$ and the remaining column vectors and scalar and then use the standard method for inverting a partitioned matrix you find that

$$
\hat{s}_{i+1}=\alpha \hat{s}_{i}+\frac{\alpha\left(S_{i i}-\Gamma_{i}\right)}{\sigma_{i}^{2}+S_{i i}-\Gamma_{i}}\left(y_{i}-\hat{s}_{i}\right),
$$

where $\Gamma_{i}=S^{T}(S+N)^{-1} S$ and $\sigma_{i}$ is the measurement error for $y_{i}$. To complete the identification with Kelly et al. (2009), we note that the variance between the data point $y_{i}$ and the 
prediction $\hat{s}_{i}$ is

$$
\left\langle\left(y_{i}-\hat{s}_{i}\right)^{2}\right\rangle=\sigma_{i}^{2}+C_{i i}-\Gamma_{i} \equiv \sigma_{i}^{2}+\Omega_{i}
$$

so Kelly et al. (2009)'s $\Omega_{i}=C_{i i}-\Gamma_{i}$. Note that Equations (A10) and (A16) differ because $\hat{s}_{i}$ in Equation (A13) does not depend on the data point $y_{i}$, but it does depend on that data point in Equation (A10). Making this substitution, we recover the forecasting expression from Kelly et al. (2009) where

$$
\hat{s}_{i+1}=\alpha \hat{s}_{i}+\frac{\alpha \Omega_{i}}{\sigma_{i}^{2}+\Omega_{i}}\left(y_{i}-\hat{s}_{i}\right)
$$

Similarly one can work out the variance in $\hat{s}_{i+1}$ to find that

$$
\Gamma_{i+1}=\alpha^{2}\left(\Gamma_{i}+\frac{\left(C_{i i}-\Gamma_{i}\right)^{2}}{\sigma_{i}^{2}+C_{i i}-\Gamma_{i}}\right)
$$

which then gives the second of the forecasting equations in Kelly et al. (2009),

$$
\Omega_{i+1}=\Omega_{0}\left(1-\alpha^{2}\right)+\alpha^{2} \Omega_{i}\left(1-\frac{\Omega_{i}}{\sigma_{i}^{2}+\Omega_{i}}\right)
$$

where $\Omega_{0}=C_{i i}$ is the diagonal element of the covariance matrix. Kelly et al. (2009) initialized their predicted time series at the process mean, while the PRH approach would initialize it to the first measurement. For this forecasting approach, the likelihood of the data given any parameters is then

$$
P(\mathbf{y} \mid \mathbf{p}) \propto \Pi_{i}\left(\Omega_{i}+\sigma_{i}^{2}\right)^{-1 / 2} \exp \left(-\frac{\left(y_{i}-\hat{s}_{i}\right)^{2}}{2\left(\Omega_{i}+\sigma_{i}^{2}\right)}\right)
$$

instead of Equation (A8).

Having demonstrated that the Kelly et al. (2009) forecasting method can be derived from the PRH approach, we next address the relative virtues of the two methods. An apparent advantage of the forecasting approach is that it is computationally $O\left(N_{\text {data }}\right)$ to compute Equation (A20), while at first glance it is $O\left(N_{\text {data }}^{3}\right)$ to compute Equation (A8) because of the matrix inversion. This is not the case for the exponential correlation function (Equation (11) ) because its inverse is tridiagonal (Rybicki \& Press 1994). If we rewrite the matrix $(S+N)^{-1}$ in Equation (A20) as $S^{-1}\left(S^{-1}+N^{-1}\right) N^{-1}$, then for a diagonal (or even tridiagonal) noise matrix $N$, all the matrix calculations, including the determinant, in Equation (A8), can be carried out in $O\left(N_{\text {data }}\right)$ operations. The total number of operations and the complexity of the implementation is somewhat higher if one includes the steps needed to automatically marginalize over the light curve mean using the linear parameters, but this then avoids having it as a parameter which must be optimized as part of the fit. Using 
the fast method from Rybicki \& Press (1994) requires the data to be time ordered and nonoverlapping $\left(t_{1}<t_{2}<t_{3} \cdots<t_{N}\right)$ since $S$ is singular if any $t_{i} \geq t_{j}$ for $i<j$ - data points at identical (or nearly identical, in the sense that $\left|t_{i}-t_{j}\right| / \tau<<1$ ) times should be combined before applying the analysis, and some care is required to stably compute the determinants.

Balancing the computational complexity is that the PRH method is more statistically powerful because it uses all the information in the light curve simultaneously. In essence, for parameter estimation we should use all available data to predict $\hat{s}_{i+1}$ not just the preceding data. We can demonstrate this by generating Monte Carlo light curves using the exponential process, estimating the parameters using both methods and examining the relative scatters in the recovered values. We use priors of $P(\tau)=1 / \tau$ and $P(\hat{\sigma})=1 / \hat{\sigma}$, as logarithmic priors are generally standard for positive definite but otherwise scale free variables. This differs from Kelly et al. (2009), who assumed a uniform prior for $\hat{\sigma}$ and $\alpha=\exp (-\Delta t / \tau)$ where $\Delta t$ is the typical spacing of the data points.

We took the same set of 109 quasars that Kelly et al. (2009) considered and fitted them with both approaches, finding results in reasonable agreement. We then took the parameters of these best fits and generated Monte Carlo realizations of the light curves (Equation (30) with the same time sampling and measurement uncertainties of the original light curves. We then re-fitted the Monte Carlo light curves using both methods and examined the differences between the input and output parameters. The results, as shown in Figure 14, unambiguously show that the PRH method produces superior results, as would be expected from using all rather than only part of the correlation information in the data. 


\section{REFERENCES}

Alcock, C., et al. 2000, ApJ, 542, 281

Assef, R. J., et al. 2009, arXiv:0909.3849

Beaulieu, J. P., et al. 1995, A\&A, 303, 137

Becker, A. C., et al. IAU Symp., 255, 357

Belokurov, V., Evans, N. W., Le Du, Y. 2004, MNRAS, 352, 233

Bentz, M. C., Peterson, B. M.; Netzer, H., Pogge, R. W.; Vestergaard, M. 2009, ApJ, 697, 160

Bramich, D. M., et al. 2008, MNRAS, 386, 887

Brockwell, P. J. \& Davis, R. A. 2003, Introduction to Time Series and Forecasting (Springer)

Burrows, D. N., et al. 2005, Space Science Reviews, 120, 165

Cabanac, R. A., et al 2007, A\&A, 461, 813

Carone, T. E., et al. 1996, ApJ, 471, 737

Collin, S., Boisson, C., Mouchet, M., Dumont, A.-M., Coupé, S., Porquet, D., Rokaki, E. 2002, A\&A, 388, 771

Cutri, R. M., Wisniewski, W. Z., Rieke, G. H., Lebofsky, M. J. 1985, ApJ, 296, 423

Cutri, R. M., et al. 2003, The IRSA 2MASS All-Sky Point Source Catalog

de Vries, W. H., Becker, R. H., White, R. L., Loomis, C. 2005, AJ, 129, 615

Dobrzycki, A., Macri, L. M., Stanek, K. Z., \& Groot, P. J. 2003a, AJ, 125, 1330

Dobrzycki, A., Eyer, L., Stanek, K. Z., \& Macri, L. M. 2005, A\&A, 442, 495

Drake, A. J. 2006, AJ, 131, 1044

Drake, A. J., et al. 2009, ApJ, 696, 870

Eyer, L., \& Woźniak, P. R. 2001, MNRAS, 327,601

Eyer, L. 2002, Acta Astron., 52, 241

Eyer, L., \& Blake, C. 2005, MNRAS, 358, 30 
Filippenko, A. V.. Li, W. D., Treffers, R. R., Modjaz, M. 2001, ASP Conference Series Vol. 246, p.121, IAU Colloquium 183

Geha, M., et al. 2003, AJ, 125, 1

Giveon, U., Maoz, D., Kaspi, S., Netzer, H., Smith, P. S. 1999, MNRAS, 306, 637

Gorjian, V., et al. 2008, ApJ, 679, 1040

Hawkins, M. R. S. 1983, MNRAS, 202, 571

Hodapp, K. W., et al. 2004, AN, 325, 636

Holtzman, J. A., Afonso, C., \& Dolphin, A. 2006 ApJS, 166, 534

Ivezic, Z., et al. 2008, arXiv:0805.2366

Kelly, B. C., Bechtold, J., \& Siemiginowska, A. 2009, ApJ, 698, 895

Keller, S. C., Bessell, M. S., Cook, K. H., Geha, M., \& Syphers, D. 2002, AJ, 124, 2039

Kołaczkowski, Z., et al. 2006, MmSAI, 77, 336

Kozłowski, S., \& Kochanek, C. S. 2009, ApJ, 701, 508

Lacy, M., et al. 2004, ApJS, 154, 166

Meegan, C. A., Fishman, G. J., Wilson, R. B., Horack, J. M., Brock, M. N., Paciesas, W. S., Pendleton, G. N., \& Kouveliotou, C. 1992, Nature, 355, 1

Miknaitis, G., et al. 2007, ApJ, 666, 674

Nelson, B. O. 1996, ApJ, 465, 87

Peterson, B. M., et al. 2000, ApJ, 542, 161

Peterson, B. M., et al. 2002, ApJ, 581, 197

Peterson, B. M., et al. 2004, ApJ, 613, 682

Pojmański, G. 1997, Acta Astron., 47, 467

Pojmański, G. 2002, Acta Astron., 52, 397

Press, W. H., Rybicki, G. B., \& Hewitt, J. N. 1992, ApJ, 385, 404 
Press, W. H., Teukolsky, S. A., Vetterling, W. T., \& Flannery, B. P. 1992, Numerical recipes in FORTRAN.

Rengstorf, A. W., et al. 2004a, ApJ, 606, 741

Rengstorf, A. W., et al. 2004b, ApJ, 617, 184

Richards, G. T., et al. 2006, AJ, 131, 2766

Rybicki, G. B., \& Press, W. H. 1992, ApJ, 398, 169

Rybicki, G. B., \& Press, W. H. 1994, Computer, 5004

Rykoff, E. S., et al. 2005, ApJ, 631, L121

Sako, M., et al. 2008, AJ, 135, 348

Santos-Lleó, M., et al. 2001, A\&A, 369, 57

Sergeev, S. G., Doroshenko, V. T., Golubinskiy, Yu. V., Merkulova, N. I., Sergeeva, E. A. 2005, ApJ, 622, 129

Sesar, B., et al. 2007, AJ, 1352236

Soszyński, I., et al. 2004a, Acta Astron., 54, 129

Soszyński, I., et al. 2004b, Acta Astron., 54, 347

Soszyński, I., et al. 2005, Acta Astron., 55, 331

Soszyński, I., et al. 2007, Acta Astron., 57, 201

Soszyński, I. 2007, ApJ, 660, 1486

Soszyński, I., et al. 2008a, Acta Astron., 58, 153

Soszyński, I., et al. 2008b, Acta Astron., 58, 163

Soszyński, I., et al. 2009, Acta Astron., 59, 1

Sterken, C., \& Jaschek, C. 1996, Book: Light Curves of Variable Stars, Cambridge University Press

Stern, D., et al. 2005, ApJ, 631, 163

Stirpe, G. M., et al. 1994, ApJ, 425, 609 
Sumi, T., et al. 2003, ApJ, 591, 43

Sumi, T., et al. 2005, MNRAS, 356, 331

Szymański, M. K., 2005, Acta Astron., 55, 43

Udalski, A., Kubiak, M., \& Szymański, M. K. 1997, Acta Astron., 47, 319

Udalski, A., Szymański, M. K., Soszyński, I., \& Poleski, R. 2008, Acta Astron., 58, 69

Vanden Berk, D. E., et al. 2004, ApJ, 601, 692

Veron, P., \& Hawkins, M. R. S. 1995, A\&A, 296, 665

Vestrand, W. T., et al. 2002, Proceedings of the SPIE, 4845, 126

Vivas, A. K., et al. 2004, AJ, 127, 1158

Woźniak, P. R., et al. 2004, AJ, 127, 2436

Wyrzykowski, Ł., et al. 2003, Acta Astron., 53, 1

Wyrzykowski, Ł., Belokurov, V. 2008, AIP Conference Proceedings, Vol. 1082, pp. 201

Wyrzykowski, Ł., et al. 2009, MNRAS, 397, 1228 


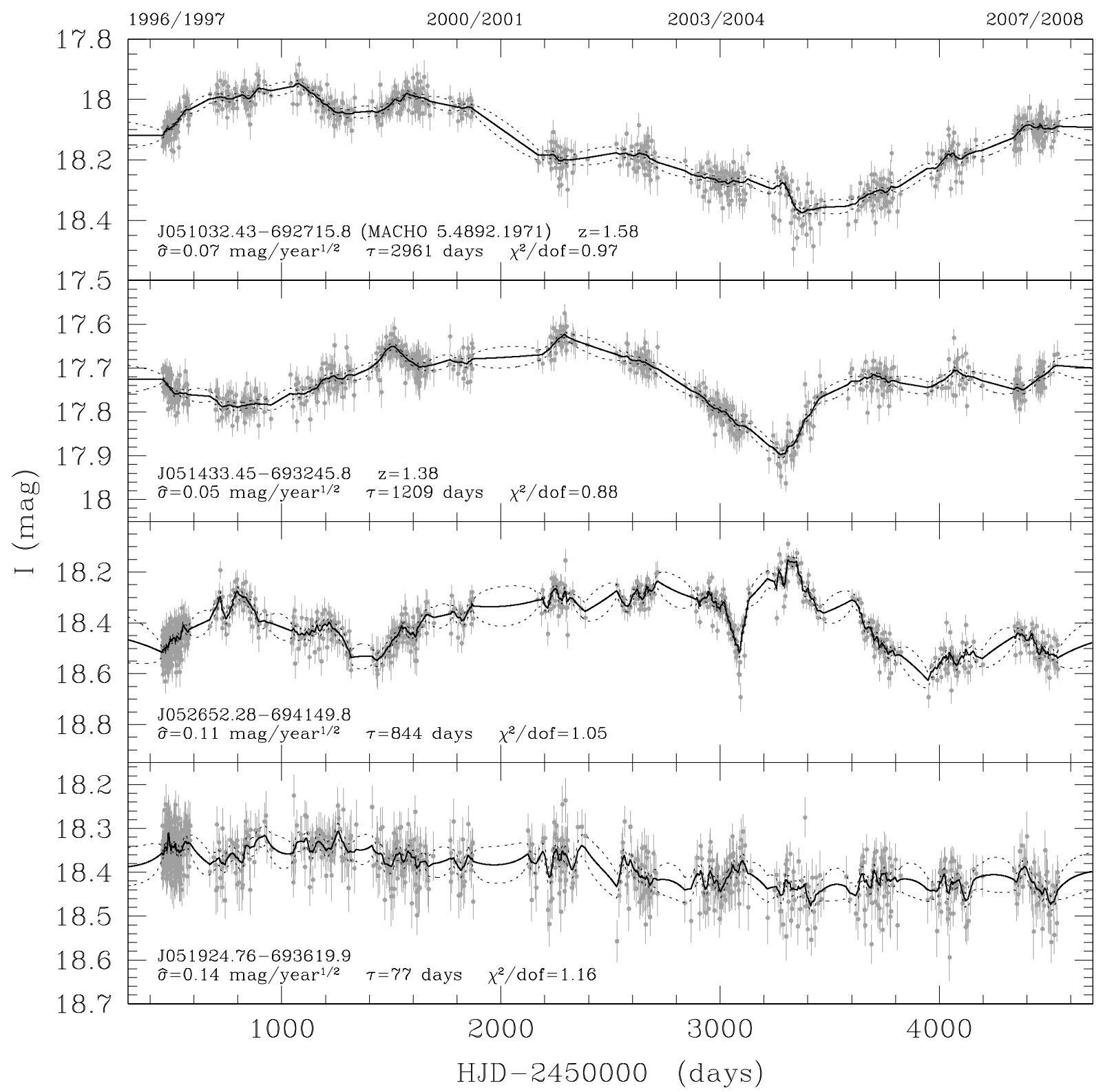

Fig. 1. - Examples of light curve models for four quasars. The 12-year-long light curves are from the OGLE-II and OGLE-III surveys (years 1997 - 2008, separated with the seasonal gaps). The top two light curves are spectroscopically confirmed quasars from Geha et al. (2003, top) and Dobrzvcki et al. (2005). The bottom two light curves are mid-IR selected quasars from the Kozłowski \& Kochanek (2009) sample (awaiting spectroscopic confirmation). The solid lines represent the best fit mean model light curves from the exponential covariance method (see appendix). The area between the dotted lines represent the $1 \sigma$ range of possible stochastic models. These "error snakes" bound the reconstructed light curve and are thinner than the data points because of the additional measurement error on the data (see the discussion of this point in Section 3). We also give the best model parameter values along with the goodness of the fit defined by the $\chi^{2}$ per degree of freedom. The average error bars on the model parameters are $\Delta \log _{10}(\tau)=0.37$ and $\Delta \log _{10}(\hat{\sigma})=0.044$. 


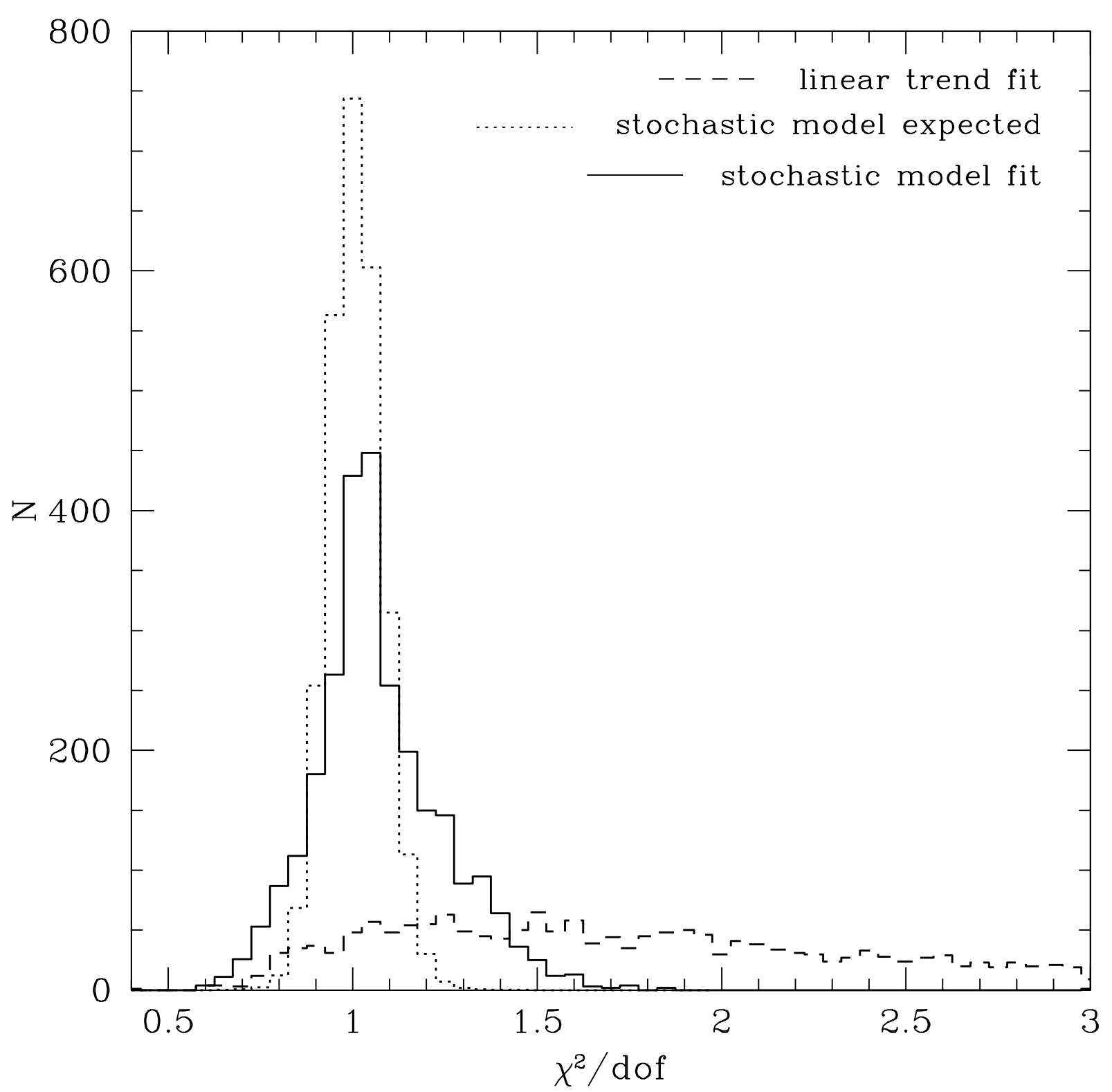

Fig. 2.- Distributions of the goodness of fit for the stochastic model (linear trend) are shown by the solid (dashed) line for the $\sim 2,700$ OGLE-III light curves of the mid-IR selected quasars from Kozłowski \& Kochanek (2009). The improvement over fitting the linear trend from using the stochastic model is clearly visible. The dotted line is the expected distribution of $\chi^{2} /$ dof based on the number of degrees of freedom for each light curve. The differences between the stochastic model and the expected distribution are some combination of errors in the estimated photometric errors, small numbers of outliers that have not been eliminated from the light curves and any poorly modeled physics. 


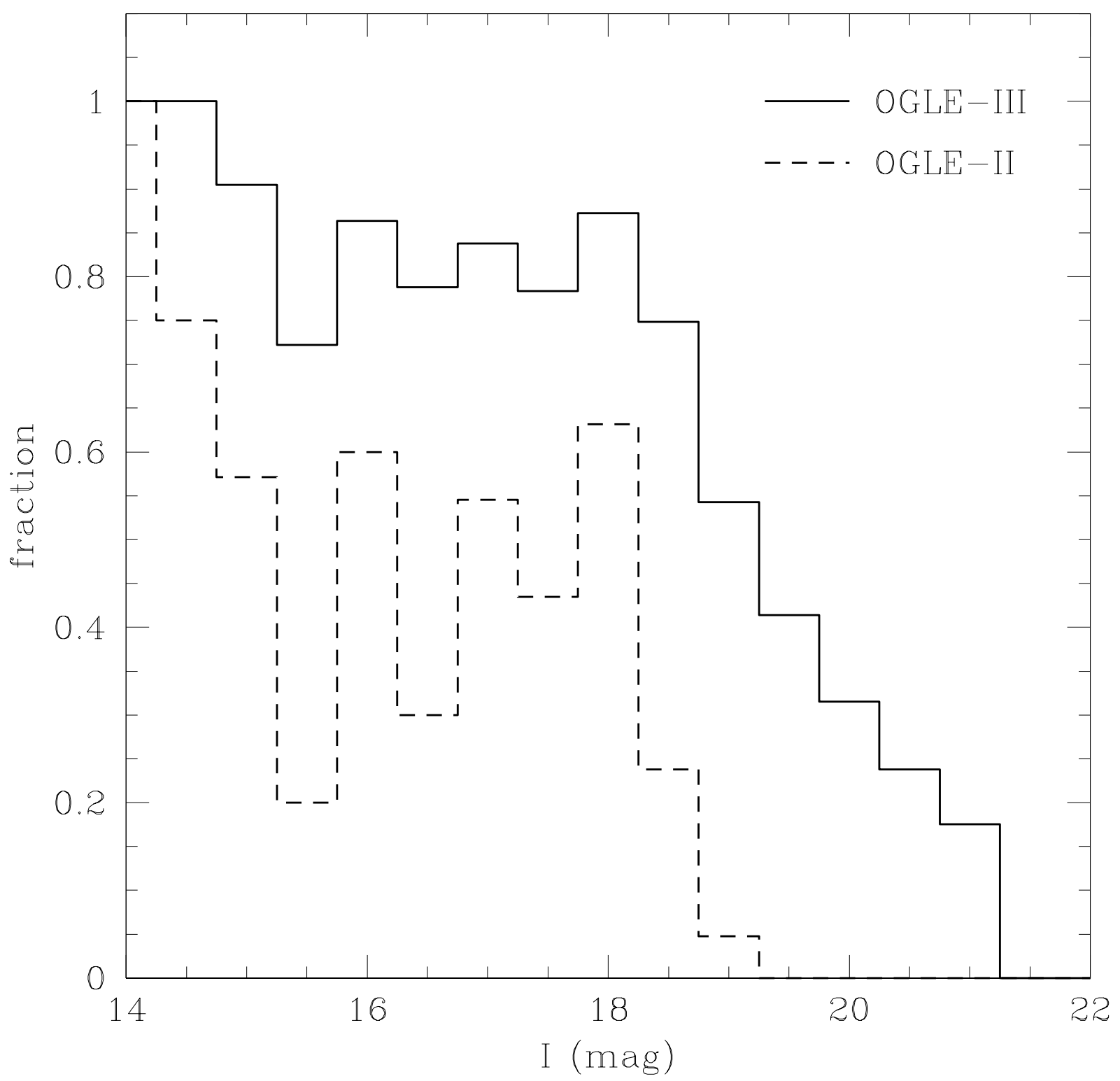

Fig. 3.- Fraction of variable QSO and YSO candidates as a function of magnitude for OGLE-III (solid line) and OGLE-II (dashed line). A source is counted as variable if it passes the variability criterion of Section 2 and has $\ln L_{\text {best }}>\ln L_{\text {noise }}+2$. 


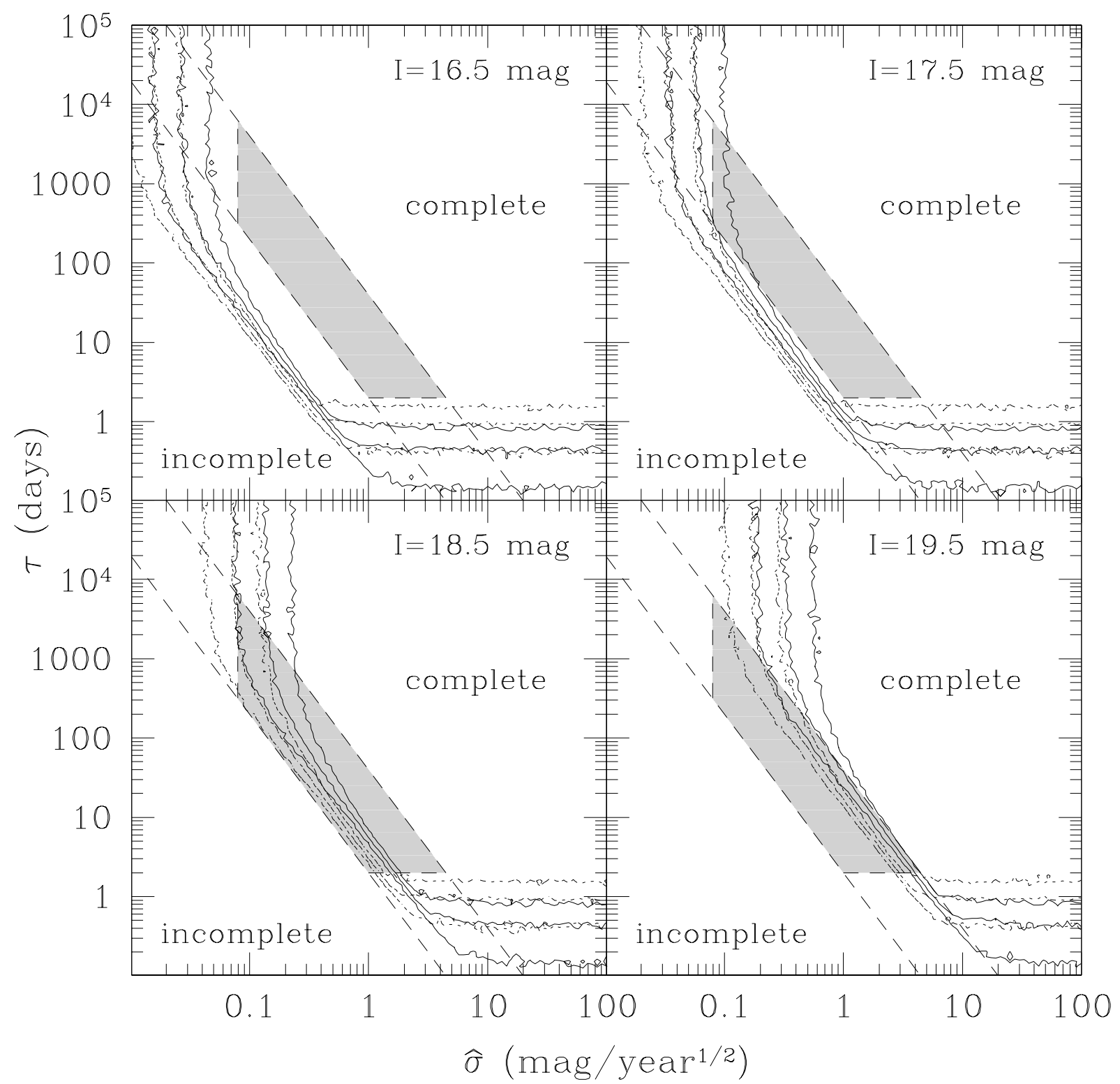

Fig. 4.- Monte Carlo simulations of completeness for source magnitudes of I = 16.5 (top left), 17.5 (top right), 18.5 (lower left) and $19.5 \mathrm{mag}$ (lower right). The OGLE-II (solid line) and OLGE-III (dotted line) surveys have different cadences, durations and depths, leading to differences in their completeness limits. While not plotted, the distributions of variable sources at a given magnitude closely track these completeness limits in the sense that variable sources passing the selection criteria are not found in the regions where the completeness calculations say they should not be detected. The three lines show completeness levels of $10 \%, 50 \%$, and $90 \%$ (from left to right). The gray shaded region shows the region occupied by quasars (Cut 2 of Section 6). 


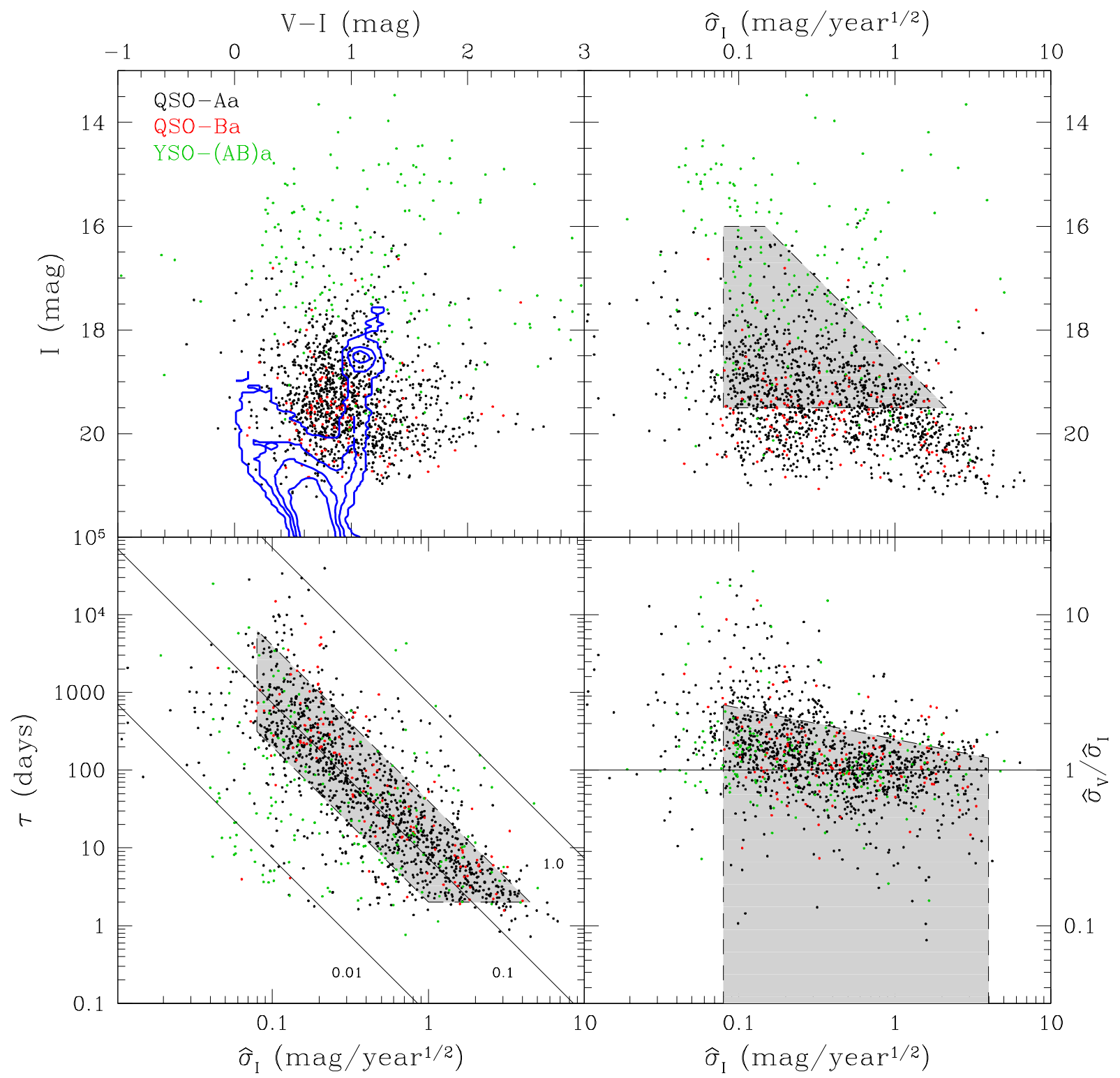

Fig. 5.- Locations of the $\sim 1,000$ variable mid-IR selected quasar candidates from Kozłowski \& Kochanek (2009) are shown in the CMD (top left), in $\hat{\sigma}_{I^{-}} I$ space (top right), in $\hat{\sigma}-\tau$ space (bottom left) and in $\left(\hat{\sigma}_{V} / \hat{\sigma}_{I}\right)-\hat{\sigma}_{I}$ space (bottom right). In the top left panel, the contours show the CMD of LMC stars, where the contours are for 1, 5, 10, and 20 stars per $0.05 \times 0.05 \mathrm{bin}$, counting from the outer contour. The RC is at $(V-I, I)=(1.05,18.3) \mathrm{mag}$. In the lower left panel we show the lines of constant asymptotic variability $\sigma$ for $0.01,0.1$, and 1.0 mag. The Kozłowski \& Kochanek (2009) candidates are coded by color, where the high purity QSO-Aa objects are black, and the high contamination QSO-Ba and YSO-(AB)a are shown in red and green, respectively. The gray shaded regions indicate the regions occupied by quasars, and are defined by Cuts of Section 6 . 


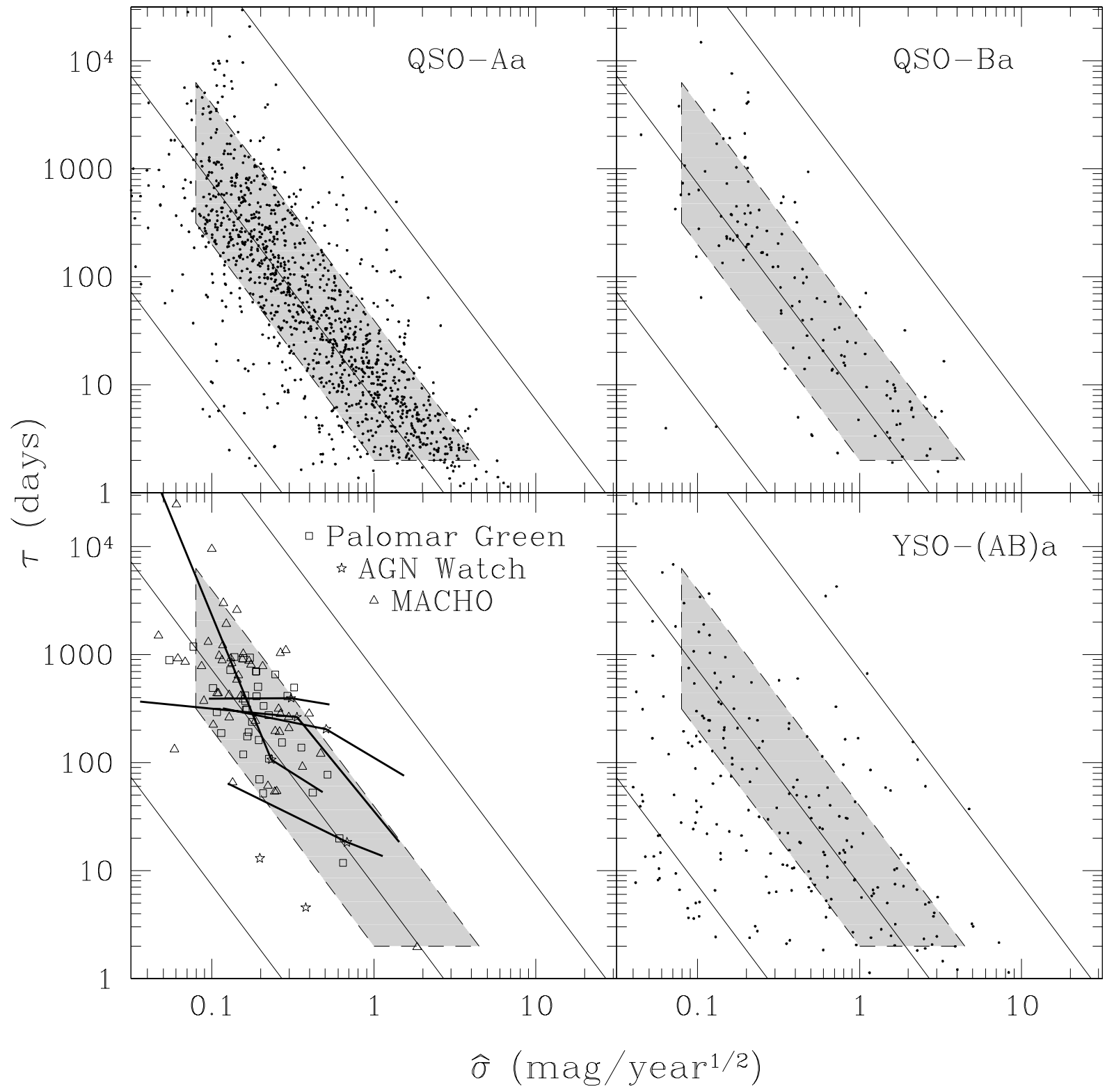

Fig. 6. - Locations of the $\sim 1,000$ variable mid-IR selected quasar candidates from Kozłowski \& Kochanek (2009) and $\sim 100$ quasars from Kelly et al. (2009) are shown in $\hat{\sigma}-\tau$ space. The QSO-Aa objects are presented in the top left panel, QSO-Ba in the top right panel, YSO-(AB)a in the bottom right panel, and the Kelly et al. (2009) quasars in the bottom left panel. The latter sample is split into Palomar Green (square), AGN Watch (star) and MACHO (triangle) quasars. We also show the lines of constant asymptotic variability $\sigma$ for $0.01,0.1$, and $1.0 \mathrm{mag}$ (from left to right) and the region occupied by quasars (Cut 2 of Section 6, gray area). The thick solid lines in the bottom-left panel are for five AGN Watch quasars, and show the change in the model parameters after adding (subtracting) the host galaxy flux outside (inside) the spectroscopic aperture used for the light curve. Adding more host moves the source to longer time scales and lower amplitudes. 


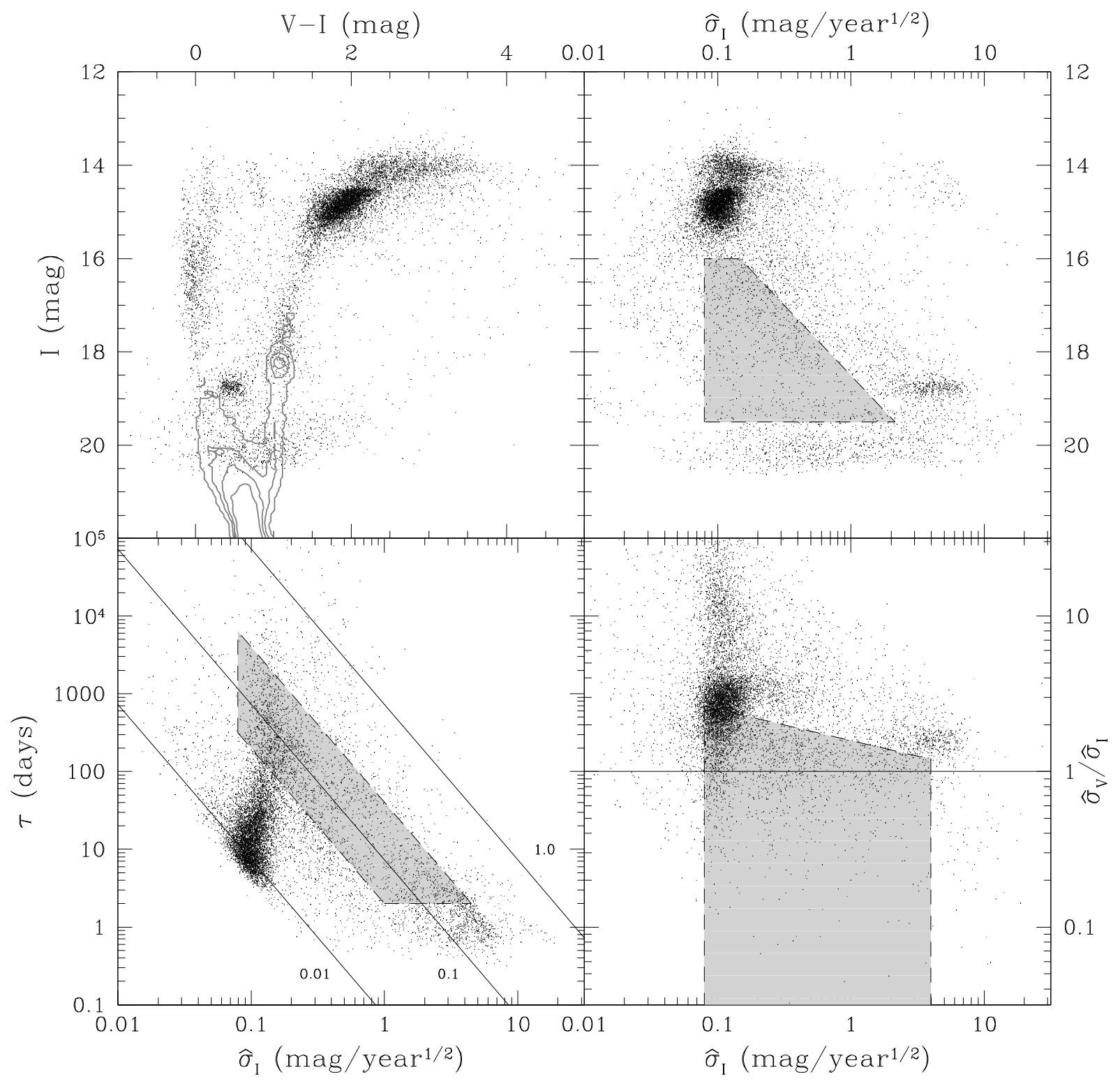

Fig. 7.- Our cleaned sample of $\sim 10,000$ variable objects from the six central OGLE-II LMC fields (SC1 - SC6) satisfying the basic variability criteria from Section 2. Panels are the same as in Figure 5 up to changes in the scales. The shaded regions show the quasar selection Cuts 2-4 from Section 6 . 


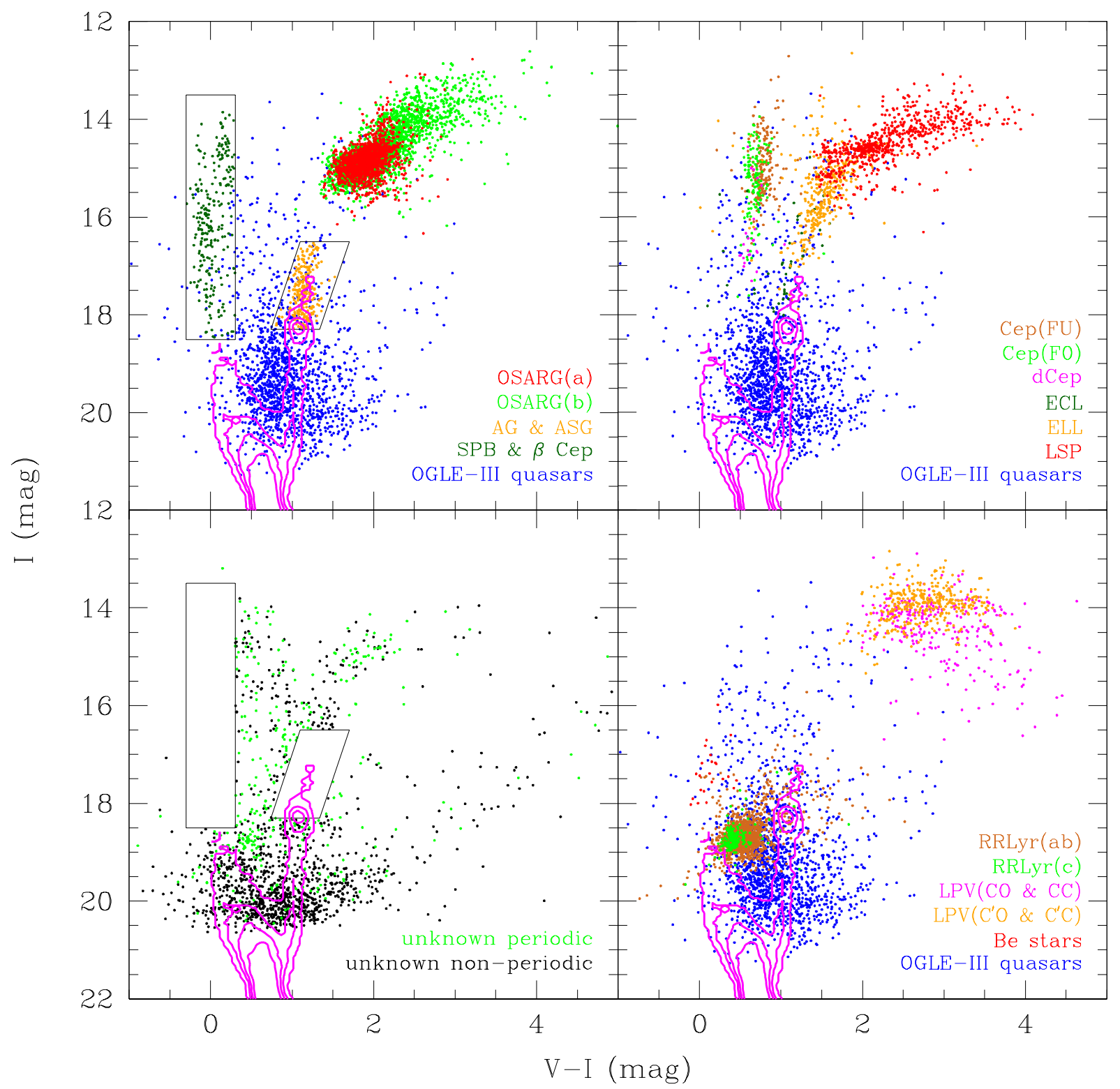

Fig. 8.- CMDs by variable type. In four panels, for clarity, we show distinct classes of variable objects. For a detailed description and discussion see text (Section 5). Objects in the two masked areas in the left panels were moved from the bottom panel to the upper one. These objects were previously not cataloged by OGLE, but they are known variable classes. In the bottom-left panel, we show both periodic and non-periodic objects of unknown origin (or previously overlooked) and in the text we discuss the fraction that are quasars. The confirmed quasars and the OGLE-II quasar candidates have a similar distribution to the OGLE-III mid-IR selected quasar candidates (blue). For clarity, we do not include these smaller quasar samples here or in the subsequent figures. 


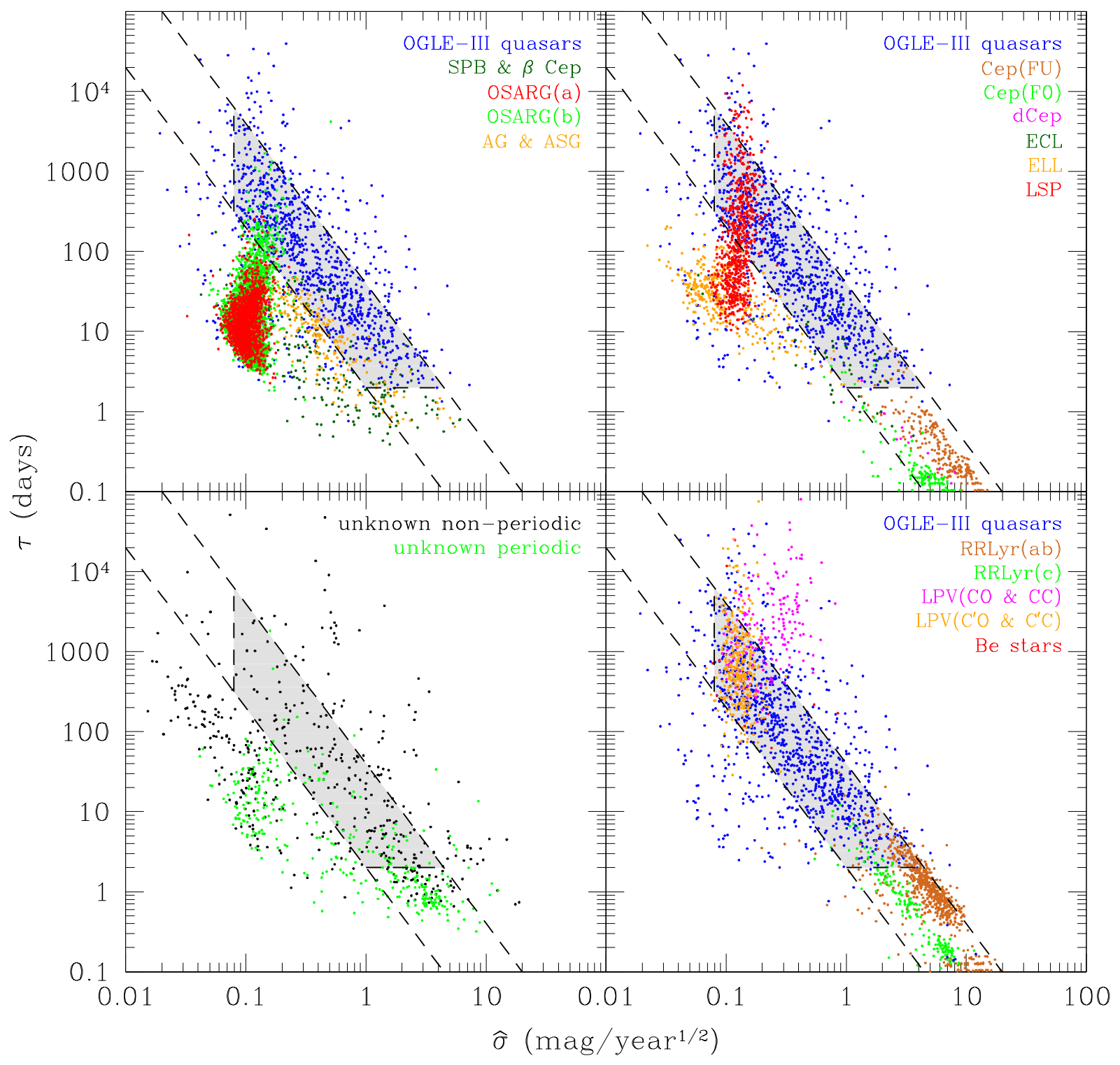

Fig. 9.- Characteristic time as a function of modified amplitude $(\hat{\sigma}-\tau)$. The four panels show the same variability classes as in Figure 8. The gray band is the quasar locus (Cut 2 in Section 6) used to separate quasars from the unknown non-periodic objects shown in the bottom-left panel. 


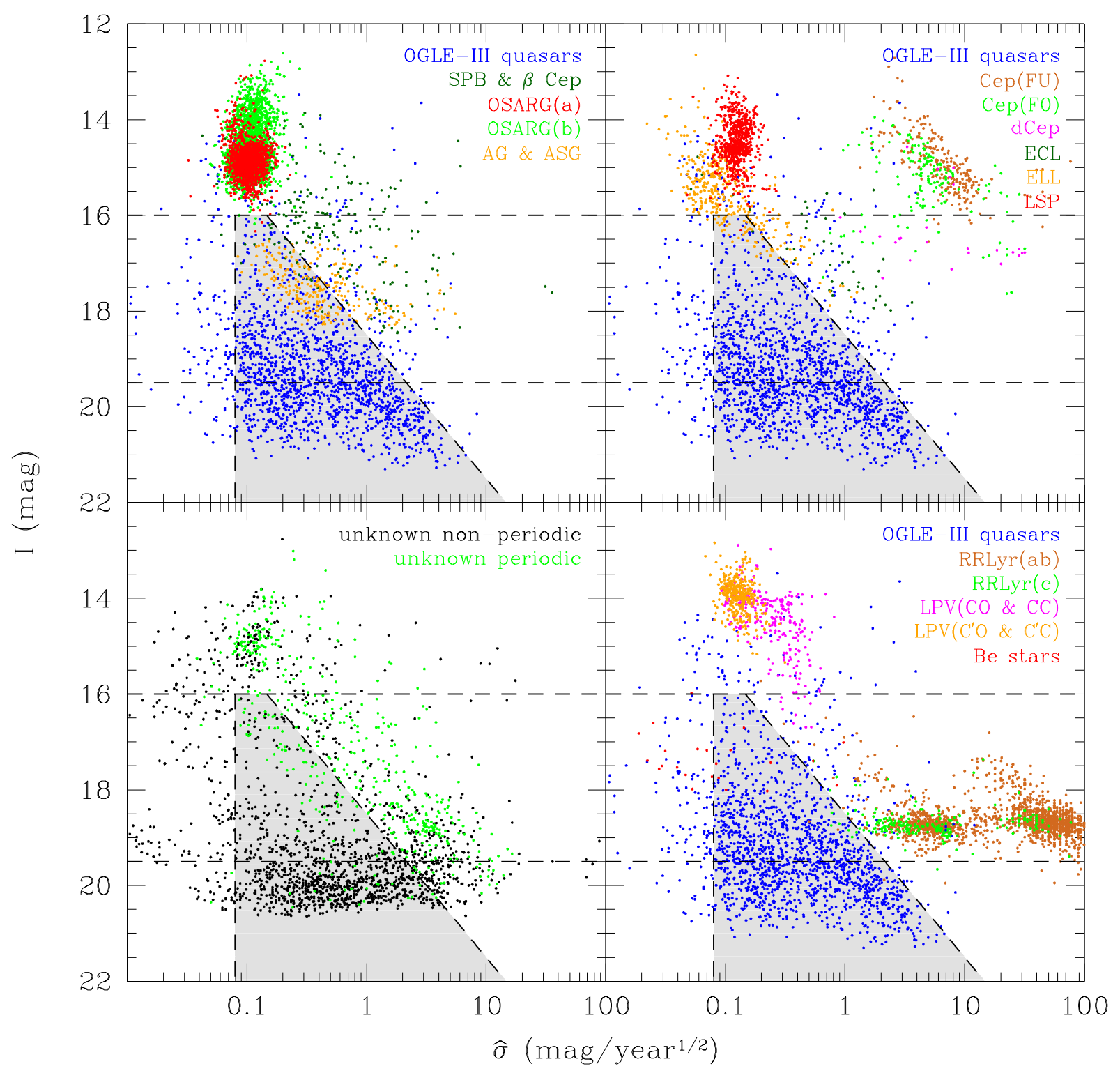

Fig. 10.- Modified amplitude vs. magnitude $(\hat{\sigma}-\mathrm{I})$. The four panels show the same variability classes as in Figure 8. The gray area is the region defined by a high density of OGLE-III quasars, which separates them from other variability classes (Cut 3 in Section 6). The heaviest contamination comes from the active giants and subgiants (AG/ASG, top left), and hot blue pulsators (SPB/ $\beta$ Cep, top left) but these can be ruled out based on their periods (see Figure 12). The gray wedge can in principle extend to arbitrarily faint magnitudes, but must be restricted based on the survey depth. For example, OGLE-II objects fainter than $I>19.5$ mag are mostly false variables (below bottom horizontal dashed line). 


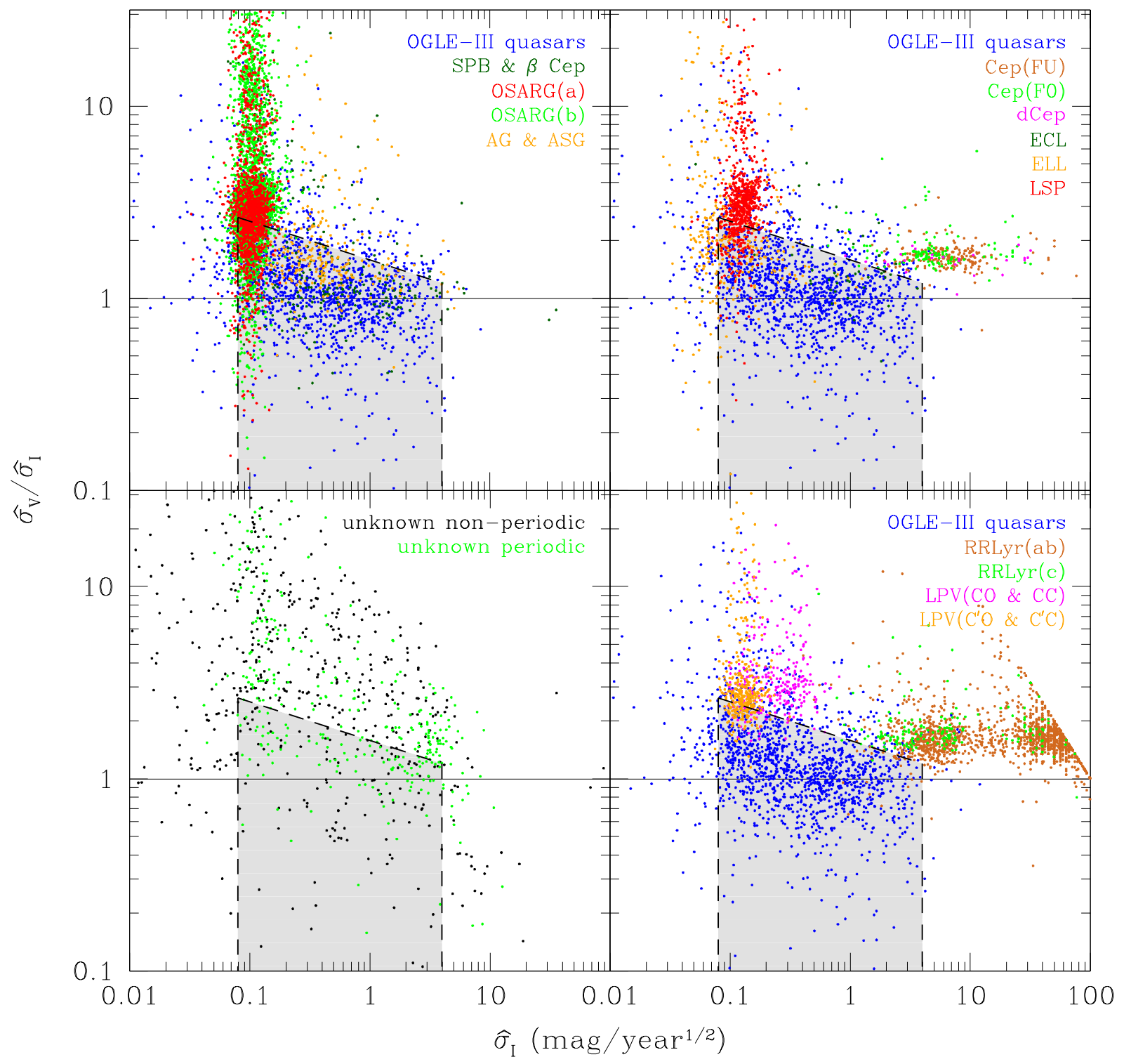

Fig. 11.- Ratio of variability amplitudes in the $V$ - and $I$-bands. It is clear that stellar objects generally vary more in the $V$-band than in the $I$-band, while QSOs show smaller differences. The gray area is the region described by Cut 4 of Section 6 . 


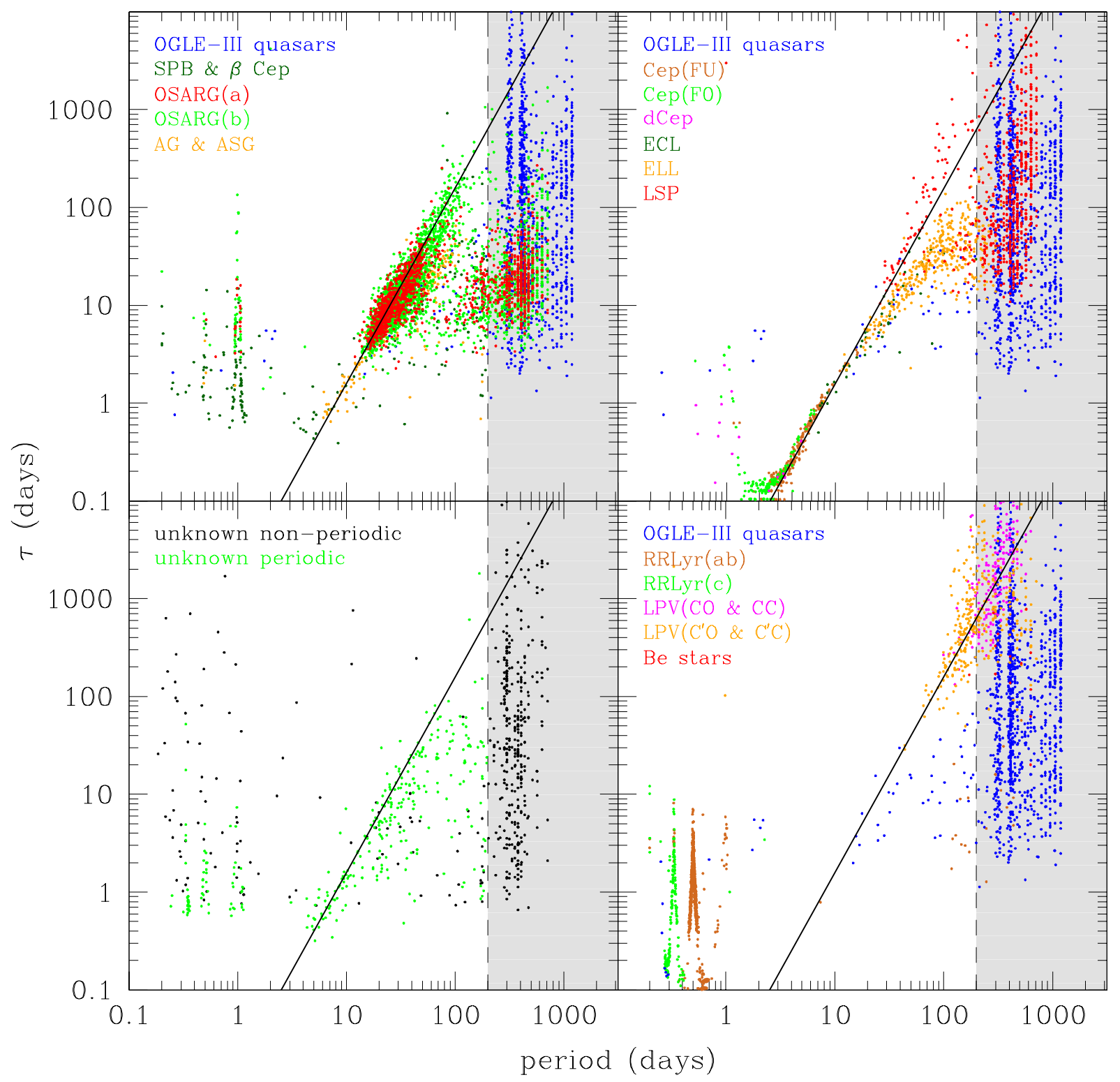

Fig. 12.- Relation between Fourier periods $P$ and time-scales $\tau$. These are not fundamental and depend on the light curve sampling, particularly on the mean epoch spacing. Except for the bottom left panel, the panels show objects with a high probability of being periodic $\left(\log _{10}\left(p_{\text {periodic }}\right)<-3\right)$ and non-periodic quasar candidates (blue dots, here selected with $\left.\log _{10}\left(p_{\text {periodic }}\right)>-3\right)$. Many periodic variable objects show a clear correlation of the characteristic time-scale $\tau$ with period $P$. These are the Cepheids, OSARGs and long period variables. Quasars clearly show no such dependence. The bifurcation in period for periodic objects at $\tau \geq 20$ days (top panels) is probably due to the multi-mode pulsations of OSARGs and LSPs. The solid line corresponds to a $\tau$-period relation described by $\log _{10}(\tau)=2 \log _{10}(P)-1.8$. The objects in the bottom left panel are previously unrecognized or overlooked non-periodic objects (black, $\log _{10}\left(p_{\text {periodic }}\right)>-3$ or $P>200$ days) and periodic variables (green, $\log _{10}\left(p_{\text {periodic }}\right)<-3$ and $P<200$ days). Spikes at $1,1 / 2,1 / 3$ and $1 / 4$ days are due to aliasing problems in the periodograms that are not fully excluded by the narrow period notches we use to suppress most diurnal aliasing problems. 


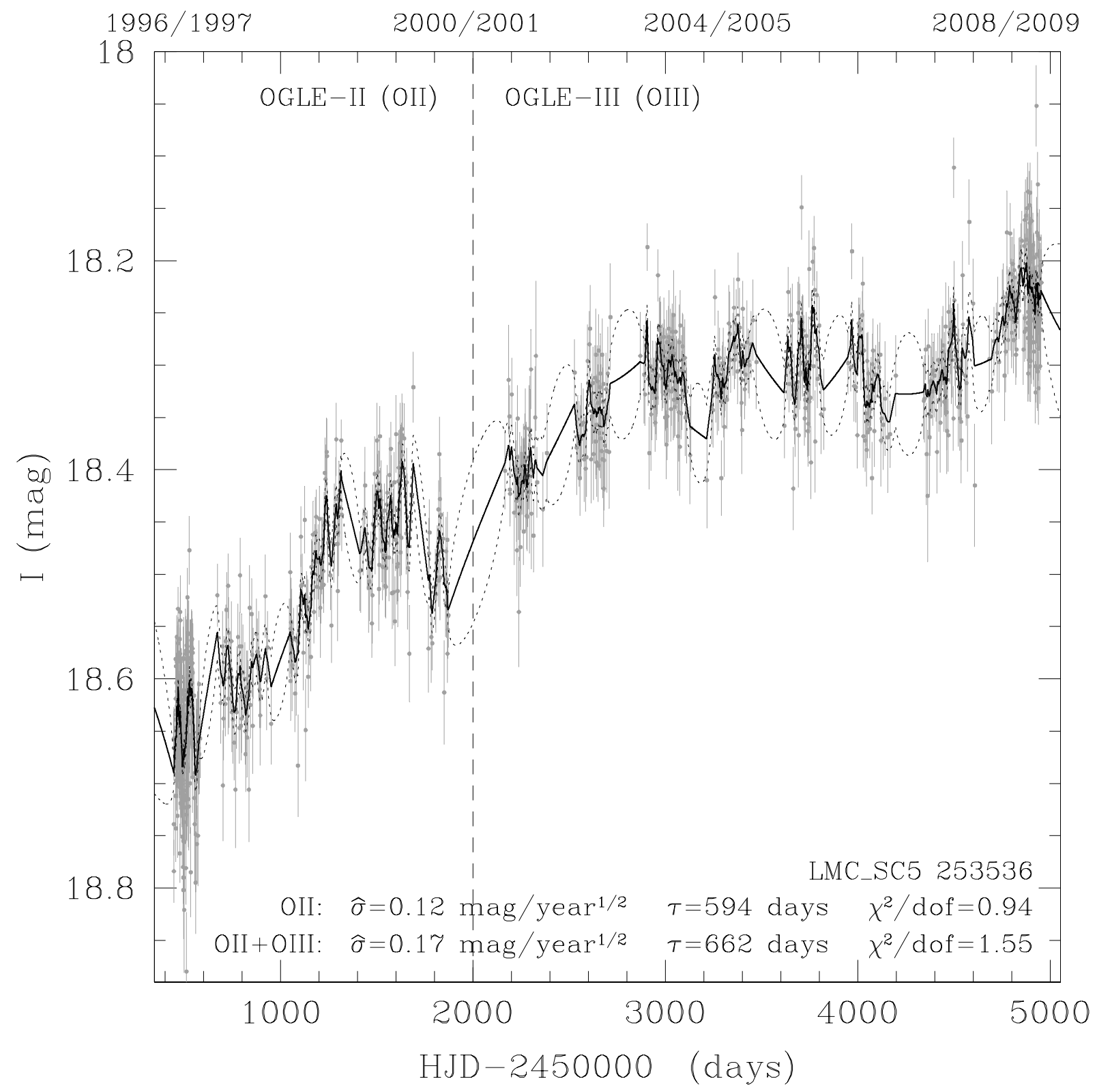

Fig. 13.- OGLE-II and OGLE-III light curve of the new variability-selected quasar candidate LMC_SC5 253536. The variability behavior of the light curve closely resembles that of quasars, and it has met the basic variability criteria of Section 2 and all four cuts from Section 6. The solid line represents the best-fit model. As in Figure 1, the area between the dotted lines represent the $1 \sigma$ range of possible stochastic models. We also give the process parameters and goodness of fit based on either the OGLE-II data or the combined data. The error bars on the model parameters are $\Delta \log _{10}(\tau)=0.49$ and $\Delta \log _{10}(\hat{\sigma})=0.089$. The vertical dashed line separates two phases of the OGLE survey. 


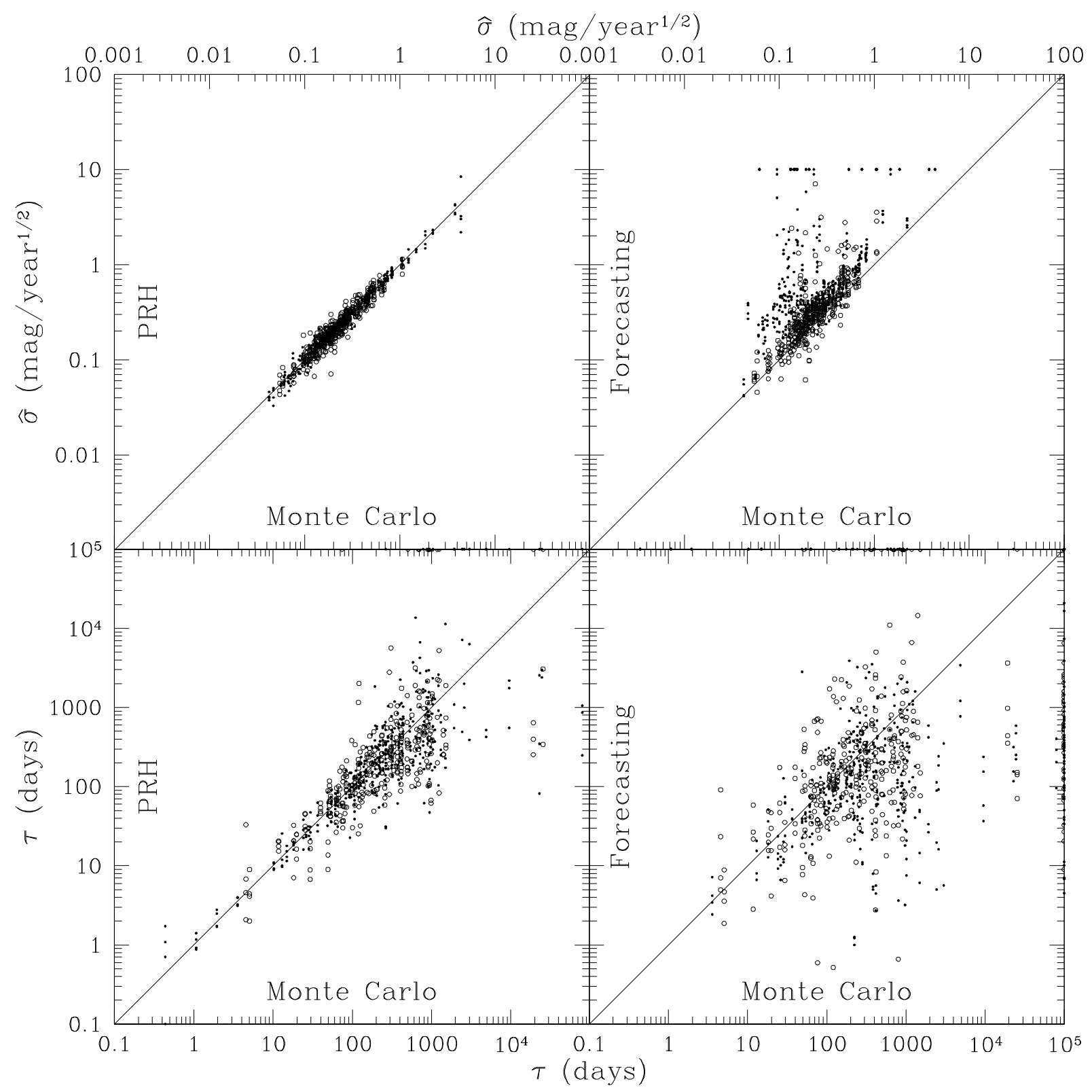

Fig. 14.- Monte Carlo comparisons of parameter estimation using the PRH and forecasting methods. For each of the 109 quasars in Kelly et al. (2009) we generated four Monte Carlo realizations of each light curve using the either the best forecasting solution (open points) or PRH solution (dots) and then refit all eight of these light curve realizations using the forecasting (right) or PRH (left) method. The greater statistical power of the PRH approach is obvious. 
Table 1. Sequential Cuts on Variable Objects.

\begin{tabular}{lcccccccc}
\hline \hline Survey & $\begin{array}{c}\text { Matched } \\
\text { to OGLE }\end{array}$ & $\begin{array}{c}\text { Object } \\
\text { Type }\end{array}$ & $\begin{array}{c}\text { Variable } \\
(>2 \sigma)\end{array}$ & Not Noise & Cut 1 \\
$p_{\text {periodic }}$ & $\begin{array}{c}\text { Cut 2 } \\
\hat{\sigma}-\tau\end{array}$ & $\begin{array}{c}\text { Cut 3 } \\
\hat{\sigma}-I\end{array}$ & $\begin{array}{c}\text { Cut } 4 \\
\hat{\sigma}_{V} / \hat{\sigma}_{I}\end{array}$ \\
\hline \multicolumn{7}{c}{ OGLE-III - Quasar Candidates } \\
\multicolumn{7}{c}{$I>16$ mag } \\
\hline OGLE-III & 2,156 & QSO-Aa & 721 & 613 & 598 & 413 & 401 & 373 \\
OGLE-III & 215 & QSO-Ba & 75 & 63 & 62 & 35 & 35 & 33 \\
OGLE-III & 198 & YSO-Aa & 147 & 140 & 116 & 47 & 28 & 27 \\
OGLE-III & 24 & YSO-Ba & 15 & 15 & 14 & 9 & 5 & 5 \\
\hline
\end{tabular}

OGLE-III - Quasar Candidates

\begin{tabular}{lcccccccc}
\multicolumn{10}{c}{$16 \leq I \leq 19.5 \mathrm{mag}$} \\
\hline OGLE-III & 2,156 & QSO-Aa & 721 & 613 & 598 & 413 & 280 & 267 \\
OGLE-III & 215 & QSO-Ba & 75 & 63 & 62 & 35 & 23 & 23 \\
OGLE-III & 198 & YSO-Aa & 147 & 140 & 116 & 47 & 26 & 25 \\
OGLE-III & 24 & YSO-Ba & 15 & 15 & 14 & 9 & 5 & 5 \\
\hline
\end{tabular}

OGLE-II - Quasar Candidates

\begin{tabular}{lcccccccc}
\multicolumn{1}{c}{$16 \leq I \leq 19.5 \mathrm{mag}$} \\
\hline OGLE-II & 209 & QSO-Aa & 23 & 23 & 19 & 8 & 6 & 4 \\
OGLE-II & 17 & QSO-Ba & 1 & 1 & 1 & 0 & 0 & 0 \\
OGLE-II & 54 & YSO-Aa & 31 & 28 & 15 & 7 & 5 & 4 \\
OGLE-II & 3 & YSO-Ba & 1 & 1 & 1 & 0 & 0 & 0 \\
\hline
\end{tabular}

OGLE-II LMC Inner Fields - All Sources

\begin{tabular}{ccccccccc}
\multicolumn{10}{c}{$16 \leq I \leq 19.5$ mag } \\
\hline OGLE-II & $I$ only & no masks & 86,301 & 64,834 & 37,599 & 8,469 & 1,939 & 731 \\
OGLE-II & $I$ only & with masks & 13,658 & 10,406 & 3,461 & 933 & 121 & 58 \\
OGLE-II & $V$ and $I$ & no masks & 71,247 & 53,942 & 30,798 & 7,033 & 1,643 & 731 \\
OGLE-II & $V$ and $I$ & with masks & 12,729 & 9,972 & 3,219 & 848 & 105 & 58 \\
\hline
\end{tabular}

OGLE-II LMC Outer Fields - All Sources

\begin{tabular}{lcccccccc}
\multicolumn{10}{c}{$16 \leq I \leq 19.5 \mathrm{mag}$} \\
\hline OGLE-II & $I$ only & no masks & 25,956 & 19,251 & 16,173 & 2,044 & 355 & 100 \\
OGLE-II & $I$ only & with masks & 2,375 & 584 & 444 & 117 & 11 & 2 \\
OGLE-II & $V$ and $I$ & no masks & 16,404 & 12,152 & 9,820 & 1,401 & 336 & 100 \\
OGLE-II & $V$ and $I$ & with masks & 1,630 & 443 & 312 & 73 & 10 & 2 \\
\hline
\end{tabular}


Table 2. Individual Cuts on Variable Objects.

\begin{tabular}{|c|c|c|c|c|c|c|c|c|}
\hline Survey & $\begin{array}{l}\text { Matched } \\
\text { to OGLE }\end{array}$ & $\begin{array}{l}\text { Object } \\
\text { Type }\end{array}$ & $\begin{array}{l}\text { Variable } \\
(>2 \sigma)\end{array}$ & Not Noise & $\begin{array}{c}\text { Cut } 1 \\
p_{\text {periodic }}\end{array}$ & $\begin{array}{l}\text { Cut } 2 \\
\hat{\sigma}-\tau\end{array}$ & $\begin{array}{c}\text { Cut } 3 \\
\hat{\sigma}-I\end{array}$ & $\begin{array}{l}\text { Cut } 4 \\
\hat{\sigma}_{V} / \hat{\sigma}_{I}\end{array}$ \\
\hline \multicolumn{9}{|c|}{$\begin{array}{c}\text { OGLE-III - Quasar Candidates } \\
I I>16 \mathrm{mag}\end{array}$} \\
\hline OGLE-III & 2,156 & QSO-Aa & 721 & 1,303 & 2,131 & 1,043 & 1,560 & 1,328 \\
\hline OGLE-III & 215 & QSO-Ba & 75 & 146 & 231 & 110 & 165 & 140 \\
\hline OGLE-III & 198 & YSO-Aa & 147 & 175 & 169 & 77 & 84 & 137 \\
\hline OGLE-III & 24 & YSO-Ba & 15 & 21 & 23 & 13 & 14 & 19 \\
\hline
\end{tabular}

OGLE-III - Quasar Candidates

\begin{tabular}{lcccccccc}
\multicolumn{1}{c}{$16 \leq I \leq 19.5 \mathrm{mag}$} \\
\hline OGLE-III & 2,156 & QSO-Aa & 721 & 1,303 & 2,131 & 1,043 & 640 & 1,328 \\
OGLE-III & 215 & QSO-Ba & 75 & 146 & 231 & 110 & 60 & 140 \\
OGLE-III & 198 & YSO-Aa & 147 & 175 & 169 & 77 & 63 & 137 \\
OGLE-III & 24 & YSO-Ba & 15 & 21 & 23 & 13 & 10 & 19 \\
\hline
\end{tabular}

OGLE-II - Quasar Candidates

\begin{tabular}{lcccccccc}
\multicolumn{10}{c}{$16 \leq I \leq 19.5 \mathrm{mag}$} \\
\hline OGLE-II & 209 & QSO-Aa & 23 & 148 & 199 & 50 & 138 & 69 \\
OGLE-II & 17 & QSO-Ba & 1 & 10 & 17 & 0 & 7 & 4 \\
OGLE-II & 54 & YSO-Aa & 31 & 48 & 37 & 15 & 21 & 19 \\
OGLE-II & 3 & YSO-Ba & 1 & 3 & 3 & 0 & 2 & 1 \\
\hline
\end{tabular}

OGLE-II LMC Inner Fields - All Sources

\begin{tabular}{|c|c|c|c|c|c|c|c|c|}
\hline OGLE-II & I only & no masks & 86,301 & 64,834 & 37,599 & 14,357 & 6,314 & 14,312 \\
\hline OGLE-II & $I$ only & with masks & 13,658 & 10,406 & 3,461 & 1,980 & 800 & 5,433 \\
\hline OGLE-II & $V$ and $I$ & no masks & 71,247 & 53,942 & 30,798 & 12,182 & 5,333 & 14,312 \\
\hline OGLE-II & $V$ and $I$ & with masks & 12,729 & 9,973 & 3,219 & 1,853 & 706 & 5,433 \\
\hline
\end{tabular}

OGLE-II LMC Outer Fields - All Sources

\begin{tabular}{lcccccccc}
\multicolumn{10}{c}{$16 \leq I \leq 19.5 \mathrm{mag}$} \\
\hline OGLE-II & $I$ only & no masks & 25,956 & 19,251 & 16,173 & 2,508 & 968 & 2,056 \\
OGLE-II & $I$ only & with masks & 3,446 & 1,272 & 658 & 171 & 167 & 298 \\
OGLE-II & $V$ and $I$ & no masks & 16,404 & 12,152 & 9,820 & 1,833 & 859 & 2,056 \\
OGLE-II & $V$ and $I$ & with masks & 1,630 & 443 & 312 & 98 & 142 & 70 \\
\hline
\end{tabular}

